\title{
GEOMETRIC COMPUTATIONAL ELECTRODYNAMICS WITH VARIATIONAL INTEGRATORS AND DISCRETE DIFFERENTIAL FORMS
}

\author{
ARI STERN, YIYING TONG, MATHIEU DESBRUN, AND JERROLD E. MARSDEN
}

\begin{abstract}
Aвstract. In this paper, we develop a structure-preserving discretization of the Lagrangian framework for electromagnetism, combining techniques from variational integrators and discrete differential forms. This leads to a general family of variational, multisymplectic numerical methods for solving Maxwell's equations that automatically preserve key symmetries and invariants.

In doing so, we demonstrate several new results, which apply both to some well-established numerical methods and to new methods introduced here. First, we show that Yee's finite-difference time-domain (FDTD) scheme, along with a number of related methods, are multisymplectic and derive from a discrete Lagrangian variational principle. Second, we generalize the Yee scheme to unstructured meshes, not just in space but in 4-dimensional spacetime. This relaxes the need to take uniform time steps, or even to have a preferred time coordinate at all. Finally, as an example of the type of methods that can be developed within this general framework, we introduce a new asynchronous variational integrator (AVI) for solving Maxwell's equations. These results are illustrated with some prototype simulations that show excellent energy and conservation behavior and lack of spurious modes, even for an irregular mesh with asynchronous time stepping.
\end{abstract}

\section{INTRODUCTION}

The Yee scheme (also known as finite-difference time-domain, or FDTD) was introduced in Yee (1966) and remains one of the most successful numerical methods used in the field of computational electromagnetics, particularly in the area of microwave problems. Although it is not a "high-order" method, it is still preferred for many applications because it preserves important structural features of Maxwell's equations that other methods fail to capture. Among these distinguishing attributes are that the Gauss constraint $\nabla \cdot \mathbf{D}=\rho$ is exactly conserved in

Date: May 27, 2009.

First author's research partially supported by a Gordon and Betty Moore Foundation fellowship at Caltech, and by NSF grant CCF-0528101.

Second and third authors' research partially supported by NSF grants CCR-0133983 and DMS0453145 and DOE contract DE-FG02-04ER25657.

Fourth author's research partially supported by NSF grant CCF-0528101. 
a discrete sense, and electrostatic solutions of the form $\mathbf{E}=-\nabla \phi$ indeed remain stationary in time (see Bondeson, Rylander, and Ingelström 2005). In this paper, we show that these desirable properties are direct consequences of the variational and discrete differential structure of the Yee scheme, which mirrors the geometry of Maxwell's equations. Moreover, we will show how to construct other variational methods that, as a result, share these same numerical properties, while at the same time applying to more general domains.

\subsection{Variational Integrators and Symmetry. Geometric numerical integrators} have been used primarily for the simulation of classical mechanical systems, where features such as symplecticity, conservation of momentum, and conservation of energy are essential. (For a survey of various methods and applications, see Hairer, Lubich, and Wanner, 2006.) Among these, variational integrators are developed by discretizing the Lagrangian variational principle of a system, and then requiring that numerical trajectories satisfy a discrete version of Hamilton's stationary-action principle. These methods are automatically symplectic, and they exactly preserve discrete momenta associated to symmetries of the Lagrangian: for instance, systems with translational invariance will conserve a discrete linear momentum, those with rotational invariance will conserve a discrete angular momentum, etc. In addition, variational integrators can be seen to display good long-time energy behavior, without artificial numerical damping (see Marsden and West 2001, for a comprehensive overview of key results).

This variational approach was extended to discretizing general multisymplectic field theories, with an application to nonlinear wave equations, in Marsden, Patrick, and Shkoller (1998) and Marsden, Pekarsky, Shkoller, and West (2001), which developed the multisymplectic approach for continuum mechanics. Building on this work, Lew, Marsden, Ortiz, and West (2003) introduced asynchronous variational integrators (AVIs), with which it becomes possible to choose a different time step size for each element of the spatial mesh, while still preserving the same variational and geometric structure as uniform-time-stepping schemes. These methods were implemented and shown to be not only practical, but in many cases superior to existing methods for problems such as nonlinear elastodynamics. Some further developments are given in Lew, Marsden, Ortiz, and West (2004).

While there have been attempts to apply the existing AVI theory to computational electromagnetics, these efforts encountered a fundamental obstacle. The key symmetry of Maxwell's equations is not rotational or translational symmetry, as in mechanics, but a differential gauge symmetry. Without taking additional 
care to preserve this gauge structure, even variational integrators cannot be expected to capture the geometry of Maxwell's equations. As will be explained, we overcome this obstacle by combining variational methods with discrete differential forms and operators. This differential/gauge structure also turns out to be important for the numerical performance of the method, and is one of the hallmarks of the Yee scheme.

1.2. Preserving Discrete Differential Structure. As motivation, consider the basic relation $\mathbf{B}=\nabla \times \mathbf{A}$, where $\mathbf{B}$ is the magnetic flux and $\mathbf{A}$ is the magnetic vector potential. Because of the vector calculus identities $\nabla \cdot \nabla \times=0$ and $\nabla \times \nabla=0$, this equation has two immediate and important consequences. First, $\mathbf{B}$ is automatically divergence-free. Second, any transformation $\mathbf{A} \mapsto \mathbf{A}+\nabla f$ has no effect on $\mathbf{B}$; this describes a gauge symmetry, for which the associated conserved momentum is $\nabla \cdot \mathbf{D}-\rho$ (which must be zero by Gauss' law). A similar argument also explains the invariance of electrostatic solutions, since $\mathbf{E}=-\nabla \phi$ is curl-free and invariant under constant shifts in the scalar potential $\phi$. Therefore, a proper variational integrator for electromagnetism should also preserve a discrete analog of these differential identities.

This can be done by viewing the objects of electromagnetism not as vector fields, but as differential forms in 4-dimensional spacetime, as is typically done in the literature on classical field theory. Using a discrete exterior calculus (called DEC) as the framework to discretize these differential forms, we find that the resulting variational integrators automatically respect discrete differential identities such as $\mathrm{d}^{2}=0$ (which encapsulates the previous div-curl-grad relations) and Stokes' theorem. Consequently, they also respect the gauge symmetry of Maxwell's equations, and therefore preserve the associated discrete momentum.

1.3. Geometry has Numerical Consequences. The Yee scheme, as we will show, is a method of precisely this type, which gives a new explanation for many of its previously observed $a$ posteriori numerical qualities. For instance, one of its notable features is that the electric field $\mathbf{E}$ and magnetic field $\mathbf{H}$ do not live at the same discrete space or time locations, but at separate nodes on a staggered lattice. The reason why this particular setup leads to improved numerics is not obvious: if we view $\mathbf{E}$ and $\mathbf{H}$ simply as vector fields in 3-space-the exact same type of mathematical object - why shouldn't they live at the same points? Indeed, many finite element method (FEM) approaches do exactly this, resulting in a "nodal" discretization. However, from the perspective of differential forms in spacetime, it becomes clear that the staggered-grid approach is more faithful to the structure of Maxwell's equations: as we will see, $\mathbf{E}$ and $\mathbf{H}$ come from objects 
that are dual to one another (the spacetime forms $F$ and $G=* F$ ), and hence they naturally live on two staggered, dual meshes.

The argument for this approach is not merely a matter of theoretical interest: the geometry of Maxwell's equations has important practical implications for numerical performance. For instance, the vector-field-based discretization, used in nodal FEM, results in spurious 3-D artifacts due to its failure to respect the underlying geometric structure. The Yee scheme, on the other hand, produces resonance spectra in agreement with theory, without spurious modes (see Bondeson et al. 2005). Furthermore, it has been shown in Haber and Ascher (2001) that staggered-grid methods can be used to develop fast numerical methods for electromagnetism, even for problems in heterogeneous media with highly discontinuous material parameters such as conductivity and permeability.

By developing a structure-preserving, geometric discretization of Maxwell's equations, not only can we better understand the Yee scheme and its characteristic advantages, but we can also construct more general methods that share its desirable properties. This family of methods includes the "Yee-like" scheme of Bossavit and Kettunen (2000), which presented the first extension of Yee's scheme to unstructured grids (e.g., simplicial meshes rather than rectangular lattices). General methods like these are highly desirable: rectangular meshes are not always practical or appropriate to use in applications where domains with curved and oblique boundaries are needed (see, for instance Clemens and Weiland 2002). By allowing general discretizations while still preserving geometry, one can combine the best attributes of the FEM and Yee schemes.

1.4. Contributions. Using DEC as a structure-preserving, geometric framework for general discrete meshes, we have obtained the following results:

(1) The Yee scheme is actually a variational integrator: that is, it can be obtained by applying Hamilton's principle of stationary action to a discrete Lagrangian.

(2) Consequently, the Yee scheme is multisymplectic and preserves discrete momentum maps (i.e., conserved quantities analogous to the continuous case of electromagnetism). In particular, the Gauss constraint is understood as a discrete momentum map of this integrator, while the preservation of electrostatic potential solutions corresponds to the identity $\mathrm{d}^{2}=0$, where $\mathrm{d}$ is the discrete exterior derivative operator.

(3) We also create a foundation for more general schemes, allowing arbitrary discretizations of spacetime, not just uniform time steps on a spatial mesh. One such scheme, introduced here, is a new asynchronous variational integrator (AVI) for Maxwell's equations, where each spatial element is 
assigned its own time step size and evolves "asynchronously" with its neighbors. This means that one can choose to take small steps where greater refinement is needed, while still using larger steps for other elements. Since refining one part of the mesh does not restrict the time steps taken elsewhere, an AVI can be computationally efficient and numerically stable with fewer total iterations. In addition to the AVI scheme, we briefly sketch how completely covariant spacetime integrators for electromagnetism can be implemented, without even requiring a $3+1$ split into space and time components.

1.5. Outline. We will begin by reviewing Maxwell's equations: first developing the differential forms expression from a Lagrangian variational principle, and next showing how this is equivalent to the familiar vector calculus formulation. We will then motivate the use of DEC for computational electromagnetics, explaining how electromagnetic quantities can be modeled using discrete differential forms and operators on a spacetime mesh. These DEC tools will then be used to set up the discrete Maxwell's equations, and to show that the resulting numerical algorithm yields the Yee and Bossavit-Kettunen schemes as special cases, as well as a new AVI method. Finally, we will demonstrate that the discrete Maxwell's equations can also be derived from a discrete variational principle, and will explore its other discrete geometric properties, including multisymplecticity and momentum map preservation.

\section{MaXwell's Equations}

This section quickly reviews the differential forms approach to electromagnetism, in preparation for the associated discrete formulation given in the next section. For more details, the reader can refer to Bossavit (1998) and Gross and Kotiuga (2004).

2.1. From Vector Fields to Differential Forms. Maxwell's equations, without free sources of charge or current, are traditionally expressed in terms of four vector fields in 3-space: the electric field $\mathbf{E}$, magnetic field $\mathbf{H}$, electric flux density $\mathbf{D}$, and magnetic flux density $\mathbf{B}$. To translate these into the language of differential forms, we begin by replacing the electric field with a 1-form $E$ and the magnetic flux density by a 2 -form $B$. These have the coordinate expressions

$$
\begin{aligned}
& E=E_{x} \mathrm{~d} x+E_{y} \mathrm{~d} y+E_{z} \mathrm{~d} z \\
& B=B_{x} \mathrm{~d} y \wedge \mathrm{d} z+B_{y} \mathrm{~d} z \wedge \mathrm{d} x+B_{z} \mathrm{~d} x \wedge \mathrm{d} y,
\end{aligned}
$$


where $\mathbf{E}=\left(E_{x}, E_{y}, E_{z}\right)$ and $\mathbf{B}=\left(B_{x}, B_{y}, B_{z}\right)$. The motivation for choosing $E$ as a 1 -form and $B$ as a 2-form comes from the integral formulation of Faraday's law,

$$
\oint_{C} \mathbf{E} \cdot \mathrm{d} \mathbf{l}=-\frac{\mathrm{d}}{\mathrm{d} t} \int_{S} \mathbf{B} \cdot \mathrm{d} \mathbf{A}
$$

where $\mathbf{E}$ is integrated over curves and $\mathbf{B}$ is integrated over surfaces. Similarly, Ampère's law,

$$
\oint_{C} \mathbf{H} \cdot \mathrm{d} \mathbf{l}=\frac{\mathrm{d}}{\mathrm{d} t} \int_{S} \mathbf{D} \cdot \mathrm{d} \mathbf{A},
$$

integrates $\mathbf{H}$ over curves and $\mathbf{D}$ over surfaces, so we can likewise introduce a 1-form $H$ and a 2-form $D$.

Now, $\mathbf{E}$ and $\mathbf{B}$ are related to $\mathbf{D}$ and $\mathbf{H}$ through the usual constitutive relations

$$
\mathbf{D}=\epsilon \mathbf{E}, \quad \mathbf{B}=\mu \mathbf{H} .
$$

As shown in Bossavit and Kettunen 2000), we can view $\epsilon$ and $\mu$ as corresponding to Hodge operators $*_{\epsilon}$ and $*_{\mu}$, which map the 1-form "fields" to 2-form "fluxes" in space. Therefore, this is compatible with viewing $E$ and $H$ as 1-forms, and $D$ and $B$ as 2-forms.

Note that in a vacuum, with $\epsilon=\epsilon_{0}$ and $\mu=\mu_{0}$ constant, one can simply express the equations in terms of $\mathbf{E}$ and $\mathbf{B}$, choosing appropriate geometrized units such that $\epsilon_{0}=\mu_{0}=c=1$, and hence ignoring the distinction between $\mathbf{E}$ and $\mathbf{D}$ and between $\mathbf{B}$ and $\mathbf{H}$. This is typically the most familiar form of Maxwell's equations, and the one that most students of electromagnetism first encounter. In this presentation, we will restrict ourselves to the vacuum case with geometrized units; for geometric clarity, however, we will always distinguish between the 1 -forms $E$ and $H$ and the 2-forms $D$ and $B$.

Finally, we can incorporate free sources of charge and current by introducing the charge density 3-form $\rho \mathrm{d} x \wedge \mathrm{d} y \wedge \mathrm{d} z$, as well as the current density 2-form $J=J_{x} \mathrm{~d} y \wedge \mathrm{d} z+J_{y} \mathrm{~d} z \wedge \mathrm{d} x+J_{z} \mathrm{~d} x \wedge \mathrm{d} y$. These are required to satisfy the continuity of charge condition $\partial_{t} \rho+\mathrm{d} J=0$, which can be understood as a conservation law (in the finite volume sense).

2.2. The Faraday and Maxwell 2-Forms. In Lorentzian spacetime, we can now combine $E$ and $B$ into a single object, the Faraday 2-form

$$
F=E \wedge \mathrm{d} t+B
$$

There is a theoretical advantage to combining the electric field and magnetic flux into a single spacetime object: this way, electromagnetic phenomena can be described in a relativistically covariant way, without favoring a particular split of spacetime into space and time components. In fact, we can turn the previous 
construction around: take $F$ to be the fundamental object, with $E$ and $B$ only emerging when we choose a particular coordinate frame. Taking the Hodge star of $F$, we also get a dual 2-form

$$
G=* F=H \wedge \mathrm{d} t-D,
$$

called the Maxwell 2-form. The equation $G=* F$ describes the dual relationship between $E$ and $B$ on one hand, and $D$ and $H$ on the other, that is expressed in the constitutive relations.

2.3. The Source 3-Form. Likewise, the charge density $\rho$ and current density $J$ can be combined into a single spacetime object, the source 3 -form

$$
\mathscr{J}=J \wedge \mathrm{d} t-\rho .
$$

Having defined $\mathscr{J}$ in this way, the continuity of charge condition simply requires that $\mathscr{J}$ be closed, i.e., $\mathrm{d} \mathscr{J}=0$.

2.4. Electromagnetic Variational Principle. Let $A$ be the electromagnetic potential 1-form, satisfying $F=\mathrm{d} A$, over the spacetime manifold $X$. Then define the 4-form Lagrangian density

$$
\mathscr{L}=-\frac{1}{2} \mathrm{~d} A \wedge * \mathrm{~d} A+A \wedge \mathscr{J}
$$

and its associated action functional

$$
S[A]=\int_{X} \mathscr{L}
$$

Now, take a variation $\alpha$ of $A$, where $\alpha$ vanishes on the boundary $\partial X$. Then the variation of the action functional along $\alpha$ is

$$
\begin{aligned}
\mathbf{d} S[A] \cdot \alpha & =\left.\frac{\mathrm{d}}{\mathrm{d} \epsilon}\right|_{\epsilon=0} S[A+\epsilon \alpha] \\
& =\int_{X}(-\mathrm{d} \alpha \wedge * \mathrm{~d} A+\alpha \wedge \mathscr{J}) \\
& =\int_{X} \alpha \wedge(-\mathrm{d} * \mathrm{~d} A+\mathscr{J}),
\end{aligned}
$$

where in this last equality we have integrated by parts, using the fact that $\alpha$ vanishes on the boundary. Hamilton's principle of stationary action requires this variation to be equal to zero for arbitrary $\alpha$, thus implying the electromagnetic Euler-Lagrange equation,

$$
\mathrm{d} * \mathrm{~d} A=\mathscr{J} .
$$

2.5. Variational Derivation of Maxwell's Equations. Since $G=* F=* \mathrm{~d} A$, then clearly Equation 2.1 is equivalent to $\mathrm{d} G=\mathscr{J}$. Furthermore, since $\mathrm{d}^{2}=0$, it follows 
that $\mathrm{d} F=\mathrm{d}^{2} A=0$. Hence, Maxwell's equations with respect to the Maxwell and Faraday 2-forms can be written as

$$
\begin{aligned}
& \mathrm{d} F=0 \\
& \mathrm{~d} G=\mathscr{J}
\end{aligned}
$$

Suppose now we choose the standard coordinate system $(x, y, z, t)$ on Minkowski space $X=\mathbb{R}^{3,1}$, and define $E$ and $B$ through the relation $F=E \wedge \mathrm{d} t+B$. Then a straightforward calculation shows that Equation 2.2 is equivalent to

$$
\begin{aligned}
\nabla \times \mathbf{E}+\partial_{t} \mathbf{B} & =0 \\
\nabla \cdot \mathbf{B} & =0 .
\end{aligned}
$$

Likewise, if $G=* F=H \wedge \mathrm{d} t-D$, then Equation 2.3 is equivalent to

$$
\begin{aligned}
\nabla \times \mathbf{H}-\partial_{t} \mathbf{D} & =\mathbf{J} \\
\nabla \cdot \mathbf{D} & =\rho .
\end{aligned}
$$

Hence this Lagrangian, differential forms approach to Maxwell's equations is strictly equivalent to the more classical vector calculus formulation in smooth spacetime. However, in discrete spacetime, we will see that the differential forms version is not equivalent to an arbitrary vector field discretization, but rather implies a particular choice of discrete objects.

2.6. Generalized Hamilton-Pontryagin Principle for Maxwell's Equations. We can also derive Maxwell's equations by using a mixed variational principle, similar to the Hamilton-Pontryagin principle introduced by Yoshimura and Marsden (2006) for classical Lagrangian mechanics. To do this, we treat $A$ and $F$ as separate fields, while $G$ acts as a Lagrange multiplier, weakly enforcing the constraint $F=\mathrm{d} A$. Define the extended action to be

$$
S[A, F, G]=\int_{X}\left[-\frac{1}{2} F \wedge * F+A \wedge \mathscr{J}+(F-\mathrm{d} A) \wedge G\right] .
$$

Then, taking the variation of the action along some $\alpha, \phi, \gamma$ (vanishing on $\partial X$ ), we have

$$
\begin{aligned}
\mathbf{d} S[A, F, G] \cdot(\alpha, \phi, \gamma) & =\int_{X}[-\phi \wedge * F+\alpha \wedge \mathscr{J}+(\phi-\mathrm{d} \alpha) \wedge G+(F-\mathrm{d} A) \wedge \gamma] \\
& =\int_{X}[\alpha \wedge(\mathscr{J}-\mathrm{d} G)+\phi \wedge(G-* F)+(F-\mathrm{d} A) \wedge \gamma] .
\end{aligned}
$$

Therefore, setting this equal to zero, we get the equations

$$
\mathrm{d} G=\mathscr{J}, \quad G=* F, \quad F=\mathrm{d} A .
$$


This is precisely equivalent to Maxwell's equations, as derived above. However, this approach provides some additional insight into the geometric structure of electromagnetics: the gauge condition $F=\mathrm{d} A$ and constitutive relations $G=* F$ are explicitly included in the equations of motion, as a direct result of the variational principle.

2.7. Reducing the Equations. When solving an initial value problem, it is not necessary to use all of Maxwell's equations to evolve the system forward in time. In fact, the curl equations (2.4) and [2.6] automatically conserve the quantities $\nabla \cdot \mathbf{B}$ and $\nabla \cdot \mathbf{D}-\rho$. Therefore, the divergence equations (2.5) and 2.7) can be viewed simply as constraints on initial conditions, while the curl equations completely describe the time evolution of the system.

There are a number of ways to see why we can justify eliminating the divergence equations. A straightforward way is to take the divergence of equations (2.4) and 2.6. Since $\nabla \cdot \nabla \times=0$, we are left with

$$
\partial_{t}(\nabla \cdot \mathbf{B})=0, \quad \partial_{t}(\nabla \cdot \mathbf{D})+\nabla \cdot \mathbf{J}=\partial_{t}(\nabla \cdot \mathbf{D}-\rho)=0 .
$$

Therefore, if the divergence constraints are satisfied at the initial time, then they are satisfied for all time, since the divergence terms are constant.

Another approach is to notice that Maxwell's equations depend only on the exterior derivative $\mathrm{d} A$ of the electromagnetic potential, and not on the value of $A$ itself. Therefore, the system has a gauge symmetry: any gauge transformation $A \mapsto A+\mathrm{d} f$ leaves $\mathrm{d} A$, and hence Maxwell's equations, unchanged. Choosing a time coordinate, we can then partially fix the gauge so that the electric scalar potential $\phi=A(\partial / \partial t)=0$ (the so-called Weyl gauge or temporal gauge), and so $A$ has only spatial components. In fact, these three remaining components correspond to those of the usual vector potential A. The reduced Euler-Lagrange equations in this gauge consist only of Equation 2.6. while the remaining gauge symmetry $\mathbf{A} \mapsto \mathbf{A}+\nabla f$ yields a momentum map that automatically preserves $\nabla \cdot \mathbf{D}-\rho$ in time. Equations (2.4) and (2.5) are automatically preserved by the identity $\mathrm{d}^{2} A=0$; they are not actually part of the Euler-Lagrange equations. A more detailed exposition of these calculations will be given in Section 5.2 .

\section{Discrete Forms in Computational Electromagnetics}

In this section, we give a quick review of the fundamental objects and operations of Discrete Exterior Calculus (DEC), a structure-preserving calculus of discrete differential forms. By construction, DEC automatically preserves a number of important geometric structures, and hence it provides a fully discrete analog of the tools used in the previous section to express the differential forms version 
of Maxwell's equations. In subsequent sections, we will use this framework to formulate Maxwell's equations discretely, emulating the continuous version.

3.1. Rationale Behind DEC for Computational Electromagnetics. Modern computational electromagnetism started in the 1960s, when the finite element method (FEM), based on nodal basis functions, was used successfully to discretize the differential equations governing 2-D static problems formulated in terms of a scalar potential. Unfortunately, the initial success of the FEM approach appeared unable to carry over to 3-D problems without spurious numerical artifacts. With the introduction of edge elements in Nédélec (1980) came the realization that a better discretization of the geometric structure of Maxwell's electromagnetic theory was key to overcoming this obstacle (see Gross and Kotiuga 2004 for more historical details). Mathematical tools developed by Weyl and Whitney in the 1950s, in the context of algebraic topology, turned out to provide the necessary foundations on which robust numerical techniques for electromagnetism can be built, as detailed in Bossavit (1998).

3.2. Discrete Differential Forms and Operators. In this section, we show how to define differential forms and operators on a discrete mesh, in preparation to use this framework for computational modeling of classical fields. By construction, the calculus of discrete differential forms automatically preserves a number of important geometric structures, including Stokes' theorem, integration by parts (with a proper treatment of boundaries), the de Rham complex, Poincaré duality, Poincaré's lemma, and Hodge theory. Therefore, this provides a suitable foundation for the coordinate-free discretization of geometric field theories. In subsequent chapters, we will also use these discrete differential forms as the space of fields on which we will define discrete Lagrangian variational principles.

The particular "flavor" of discrete differential forms and operators we will be using is known as discrete exterior calculus, or DEC for short; see Hirani (2003); Leok (2004). (For related efforts in this direction, see also Harrison, 2005 and Arnold, Falk, and Winther. 2006.) Guided by Cartan's exterior calculus of differential forms on smooth manifolds, DEC is a discrete calculus developed, ab initio, on discrete manifolds, so as to maintain the covariant nature of the quantities involved. This computational tool is based on the notion of discrete chains and cochains, used as basic building blocks for compatible discretizations of important geometric structures such as the de Rham complex (Desbrun, Kanso, and Tong, 2008). The chain and cochain representations are not only attractive from a computational perspective due to their conceptual simplicity and elegance; as we will see, they 
also originate from a theoretical framework defined by Whitney (1957), who introduced the Whitney and de Rham maps that establish an isomorphism between simplicial cochains and Lipschitz differential forms.

Mesh and Dual Mesh. DEC is concerned with problems in which the smooth $n$-dimensional manifold $X$ is replaced by a discrete mesh - precisely, by a cell complex that is manifold, admits a metric, and is orientable. The simplest example of such a mesh is a finite simplicial complex, such as a triangulation of a 2-dimensional surface. We will generally denote the complex by $K$, and a cell in the complex by $\sigma$.

Given a mesh $K$, one can construct a dual mesh $* K$, where each $k$-cell $\sigma$ corresponds to a dual $(n-k)$-cell $* \sigma$. ( $* K$ is "dual" to $K$ in the sense of a graph dual.) One way to do this is as follows: place a dual vertex at the circumcenter of each $n$-simplex, then connect two dual vertices by an edge wherever the corresponding $n$-simplices share an $(n-1)$-simplex, and so on. This is called the circumcentric dual, and it has the important property that primal and dual cells are automatically orthogonal to one another, which is advantageous when defining an inner product (as we will see later in this section). For example, the circumcentric dual of a Delaunay triangulation, with the Euclidean metric, is its corresponding Voronoi diagram (see Figure 1). For more on the dual relationship between Delaunay triangulations and Voronoi diagrams, a standard reference is O'Rourke (1998). A similar construction of the circumcenter can be carried out for higher-dimensional Euclidean simplicial complexes, as well as for simplicial meshes in Minkowski space. Note that, in both the Euclidean and Lorentzian cases, the circumcenter may actually lie outside the simplex if it has a very bad aspect ratio, underscoring the importance of mesh quality for good numerical results.

There are alternative ways to define the dual mesh-for example, placing dual vertices at the barycenter rather than the circumcenter-but we will use the circumcentric dual unless otherwise noted. Note that a refined definition of the dual mesh, where dual cells at the boundary are restricted to $K$, will be discussed in Section 3.3 to allow proper enforcement of boundary conditions in computational electromagnetics.

Discrete Differential Forms. The fundamental objects of DEC are discrete differential forms. A discrete $k$-form $\alpha^{k}$ assigns a real number to each oriented $k$-dimensional cell $\sigma^{k}$ in the mesh $K$. (The superscripts $k$ are not actually required by the notation, but they are often useful as reminders of what order of form or cell we are dealing with.) This value is denoted by $\left\langle\alpha^{k}, \sigma^{k}\right\rangle$, and can be 


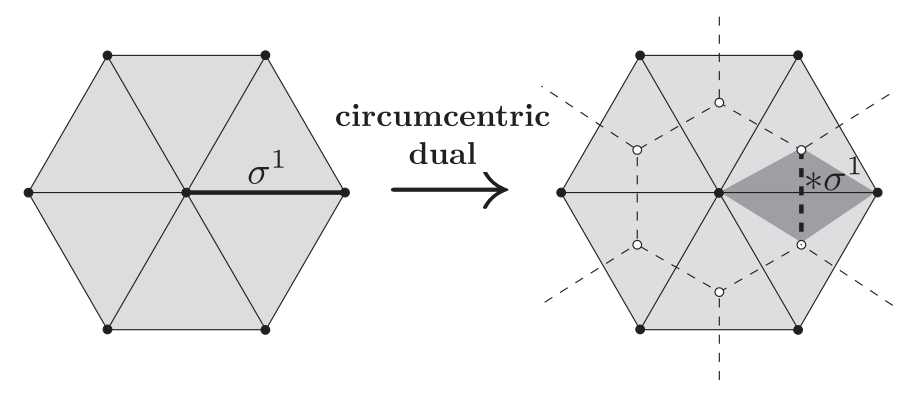

Figure 1: Given a 2-D simplicial mesh (left), we can construct its circumcentric dual mesh, called the Voronoi diagram of the primal mesh (right). In bold, we show one particular primal edge $\sigma^{1}$ (left) and its corresponding dual edge $* \sigma^{1}$ (right); the convex hull of these cells $\mathrm{CH}\left(\sigma^{1}, * \sigma^{1}\right)$ is shaded dark grey.

thought of as the value of $\alpha^{k}$ "integrated over" the element $\sigma^{k}$, i.e.,

$$
\langle\alpha, \sigma\rangle \equiv \int_{\sigma} \alpha .
$$

For example, 0 -forms assign values to vertices, 1 -forms assign values to edges, etc. We can extend this to integrate over discrete paths by linearity: simply add the form's values on each cell in the path, taking care to flip the sign if the path is oriented opposite the cell. Formally, these "paths" of $k$-dimensional elements are called chains, and discrete differential forms are cochains, where $\langle\cdot, \cdot\rangle\rangle$ is the pairing between cochains and chains.

Differential forms can be defined either on the mesh $K$ or on its dual $* K$. We will refer to these as primal forms and dual forms respectively. Note that there is a natural correspondence between primal $k$-forms and dual $(n-k)$-forms, since each primal $k$-cell has a dual $(n-k)$-cell. This is an important property that will be used below to define the discrete Hodge star operator.

Exterior Derivative. The discrete exterior derivative d is constructed to satisfy Stokes' theorem, which in the continuous sense is written

$$
\int_{\sigma} \mathrm{d} \alpha=\int_{\partial \sigma} \alpha .
$$

Therefore, if $\alpha$ is a discrete differential $k$-form, then the $(k+1)$-form $\mathrm{d} \alpha$ is defined on any $(k+1)$-chain $\sigma$ by

$$
\langle\mathrm{d} \alpha, \sigma\rangle=\langle\alpha, \partial \sigma\rangle,
$$

where $\partial \sigma$ is the $k$-chain boundary of $\sigma$. For this reason, $\mathrm{d}$ is often called the coboundary operator in cohomology theory. 
Diagonal Hodge Star. The discrete Hodge star transforms $k$-forms on the primal mesh into $(n-k)$-forms on the dual mesh, and vice-versa. In our setup, we will use the so-called diagonal (or mass-lumped) approximation of the Hodge star Bossavit 1998 because of its simplicity, but note that higher-order accurate versions can be substituted. Given a discrete form $\alpha$, its Hodge star $* \alpha$ is defined by the relation

$$
\frac{1}{|* \sigma|}\langle * \alpha, * \sigma\rangle=\kappa(\sigma) \frac{1}{|\sigma|}\langle\alpha, \sigma\rangle,
$$

where $|\sigma|$ and $|* \sigma|$ are the volumes of these elements, and $\kappa$ is the causality operator, which equals +1 when $\sigma$ is spacelike and -1 otherwise. (For more information on alternative discrete Hodge operators, the reader may refer to, e.g., Arnold et al. 2006, Auchmann and Kurz, 2006: Tarhasaari, Kettunen, and Bossavit 1999: Wang, Weiwei, Tong, Desbrun, and Schröder. 2006.)

Inner Product. Define the $\mathbb{L}^{2}$ inner product $(\cdot, \cdot)$ between two primal $k$-forms to be

$$
\begin{aligned}
(\alpha, \beta) & =\sum_{\sigma^{k}} \kappa(\sigma)\left(\begin{array}{l}
n \\
k
\end{array}\right) \frac{|\mathrm{CH}(\sigma, * \sigma)|}{|\sigma|^{2}}\langle\alpha, \sigma\rangle\langle\beta, \sigma\rangle \\
& =\sum_{\sigma^{k}} \kappa(\sigma) \frac{|* \sigma|}{|\sigma|}\langle\alpha, \sigma\rangle\langle\beta, \sigma\rangle
\end{aligned}
$$

where the sum is taken over all $k$-dimensional elements $\sigma$, and $\mathrm{CH}(\sigma, * \sigma)$ is the $n$-dimensional convex hull of $\sigma \cup * \sigma$ (see Figure 1). The final equality holds as a result of using the circumcentric dual, since $\sigma$ and $* \sigma$ are orthogonal to one another, and hence $|\mathrm{CH}(\sigma, * \sigma)|=\left(\begin{array}{l}n \\ k\end{array}\right)^{-1}|\sigma||* \sigma|$. (Indeed, this is one of the advantages of using the circumcentric dual, since one only needs to store volume information about the primal and dual cells themselves, and not about these primal-dual convex hulls.) This inner product can be expressed in terms of $\alpha \wedge * \beta$, as in the continuous case, for a particular choice of the discrete primal-dual wedge product; see Desbrun, Hirani, and Marsden (2003).

Note that since we have already defined a discrete version of the operators $\mathrm{d}$ and $*$, we immediately have a discrete codifferential $\delta$, with the same formal expression as given previously. See Figure 2 for a visual diagram of primal and dual discrete forms, along with the corresponding operators $\mathrm{d}, *, \delta$, for the case where $K$ is a 3-D tetrahedral mesh.

Implementing DEC. DEC can be implemented simply and efficiently using linear algebra. A $k$-form $\alpha$ can be stored as a vector, where its entries are the values of $\alpha$ on each $k$-cell of the mesh. That is, given a list of $k$-cells $\sigma_{i}^{k}$, the entries of the vector are $\alpha_{i}=\left\langle\alpha, \sigma_{i}^{k}\right\rangle$. The exterior derivative d, taking $k$-forms to $(k+1)$-forms, is then represented as a matrix: in fact, it is precisely the incidence matrix between 
0 -forms (vertices)
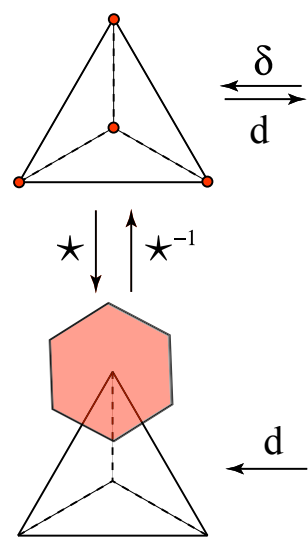

1-forms (edges)
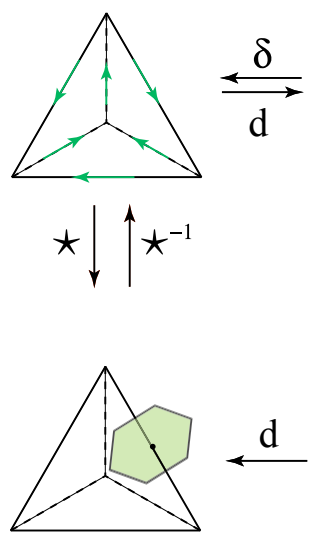

2-forms (faces)

3-forms (tets)
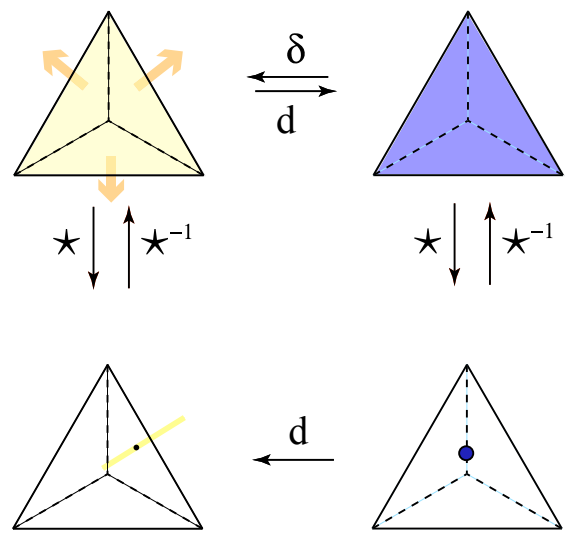

Figure 2: This figure is an illustration of discrete differential forms and operators on a 3-D simplicial mesh. In the top row, we see how a discrete $k$-form lives on $k$ cells of the primal mesh, for $k=0,1,2,3$; the bottom row shows the location of the corresponding dual $(n-k)$-forms on the dual mesh. The differential operators $\mathrm{d}$ and $\delta$ map "horizontally" between $k$ and $(k+1)$ forms, while the Hodge star $*$ and its inverse $*^{-1}$ map "vertically" between primal and dual forms.

$k$-cells and $(k+1)$-cells in the mesh, with sparse entries \pm 1 . The Hodge star taking primal $k$-forms to dual $(n-k)$-forms becomes a square matrix, and in the case of the diagonal Hodge star, it is the diagonal matrix with entries $\kappa\left(\sigma_{i}^{k}\right) \frac{\left|* \sigma_{i}^{k}\right|}{\left|\sigma_{i}^{k}\right|}$. The discrete inner product is then simply the Hodge star matrix taken as a quadratic form.

Because of this straightforward isomorphism between DEC and linear algebra, problems posed in the language of DEC can take advantage of existing numerical linear algebra codes. For more details on programming and implementation, refer to Elcott and Schröder (2005).

3.3. Initial and Boundary Values with DEC. Particular care is required to properly enforce initial and boundary conditions on the discrete spacetime boundary $\partial K$. For example, in electromagnetism, we may wish to set initial conditions for $E$ and $B$ at time $t_{0}$ - but while $B$ is defined on $\partial K$ at $t_{0}, E$ is not. In fact, as we will see, $E$ lives on edges that are extruded between the time slices $t_{0}$ and $t_{1}$, so unless we modify our definitions, we can only initialize $E$ at the half-step $t_{1 / 2}$. (This half-step issue also arises with the standard Yee scheme.) There are some applications where it may be acceptable to initialize $E$ and $B$ at separate times (for example, when the fields are initialized randomly and integrated for a long time to compute a resonance spectrum), but we wish to be able to handle the 


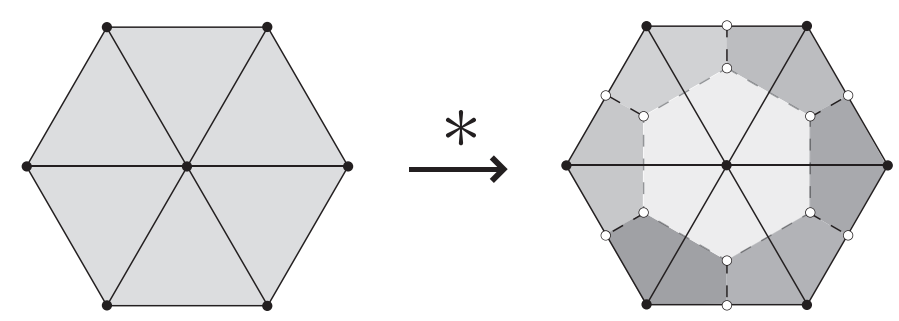

Figure 3: In this 2-D example, the dual mesh is properly defined near the boundary by adding dual vertices on the boundary edges. The restricted Voronoi cells of the primal boundary vertices (shaded at right) thus have boundaries containing both dual edges (dashed lines) and primal boundary half-edges.

more general case. Although our previous exposition of DEC thus far applies anywhere away from a boundary, notions as simple as "dual cell" need to be defined carefully on or near $\partial K$.

For a primal mesh $K$, the dual mesh $* K$ is defined as the Voronoi dual of $K$ restricted to $K$. This truncates the portion of the dual cells extending outside of $K$; compare Figure 3 with the earlier Figure 1 . This new definition results in the addition of a dual vertex at the circumcenter of each boundary $(n-1)$-simplex, in addition to the interior $n$-simplices as previously defined. To complete the dual mesh $* K$, we add a dual edge between adjacent dual vertices on the boundary, as well as between dual boundary vertices and their neighboring interior dual vertices, and proceed similarly with higher-dimensional dual cells. For intuition, one can imagine the ( $n-1)$-dimensional boundary to be a vanishingly thin $n$ dimensional shell. That is, each boundary $(k-1)$-simplex can be thought of as a prismal $k$-cell that has been "squashed flat" along the boundary normal direction. This process is quite similar to the use of "ghost cells" at the boundary, as is commonly done for finite volume methods (see LeVeque, 2002). Note that these additional dual cells provide the boundary $\partial K$ with its own dual mesh $*(\partial K)$. In fact, the boundary of the dual is now equal to the dual of the boundary, i.e., $\partial(* K)=*(\partial K)$. Returning to the example of initial conditions on $E$ and $B$, we recall that $E$ is defined on extruded faces normal to the time slice $t_{0}$. Therefore, thanks to the proper restriction of the Voronoi diagram to the domain, we can now define $E$ on edges in $\partial K$ at time $t_{0}$, where these edges can be understood as vanishingly thin faces (i.e., extruded between some $t_{-\epsilon}$ and $t_{0}$ for $\epsilon \rightarrow 0$ ). Notice finally that with this construction of $* K$, there is a dual relationship between Dirichlet conditions on the dual mesh and Neumann conditions on the primal mesh, e.g., between primal fields and dual fluxes, as expected. 
3.4. Discrete Integration by Parts with Boundary Terms. With the dual mesh properly defined, dual forms can now be defined on the boundary. Therefore, the discrete duality between $\mathrm{d}$ and $\delta$ can be generalized to include nonvanishing boundary terms. If $\alpha$ is a primal $(k-1)$-form and $\beta$ is a primal $k$-form, then

$$
(\mathrm{d} \alpha, \beta)=(\alpha, \delta \beta)+\langle\alpha \wedge * \beta, \partial K\rangle .
$$

In the boundary integral, $\alpha$ is still a primal $(k-1)$-form on $\partial K$, while $* \beta$ is an $(n-k)$-form taken on the boundary dual $*(\partial K)$. Formula $(3.1)$ is readily proved using the familiar method of discrete "summation by parts," and thus agrees with the integration by parts formula for smooth differential forms.

\section{Implementing Maxwell's Equations with DEC}

In this section, we explain how to obtain numerical algorithms for solving Maxwell's equations with DEC. To do so, we will proceed in the following order. First, we will find a sensible way to define the discrete forms $F, G$, and $\mathscr{J}$ on a spacetime mesh. Next, we will use the DEC version of the operators $\mathrm{d}$ and $*$ to obtain the discrete Maxwell's equations. While we haven't yet shown that these equations are variational in the discrete sense, we will show later in Section 5 that the Lagrangian derivation of the smooth Maxwell's equations also holds with the DEC operators, in precisely the same way. Finally, we will discuss how these equations can be used to define a numerical method for computational electromagnetics.

In particular, for a rectangular grid, we will show that our setup results in the traditional Yee scheme. For a general triangulation of space with equal time steps, the resulting scheme will be Bossavit and Kettunen's scheme. We will then develop an AVI method, where each spatial element can be assigned a different time step, and the time integration of Maxwell's equations can be performed on the elements asynchronously. Finally, we will comment on the equations for fully generalized spacetime meshes, e.g., an arbitrary meshing of $\mathbb{R}^{3,1}$ by 4 -simplices.

Note that the idea of discretizing Maxwell's equations using spacetime cochains was mentioned in, e.g., Leok (2004), as well as in a paper by Wise (2006) taking the more abstract perspective of higher-level " $p$-form" versions of electromagnetism and category theory.

4.1. Rectangular Grid. Suppose that we have a rectangular grid in $\mathbb{R}^{3,1}$, oriented along the axes $(x, y, z, t)$. To simplify this exposition (although it is not necessary), let us also suppose that the grid has uniform space and time steps $\Delta x, \Delta y, \Delta z, \Delta t$. Note that the DEC setup applies directly to a non-simplicial rectangular mesh, since an $n$-rectangle does in fact have a circumcenter. 

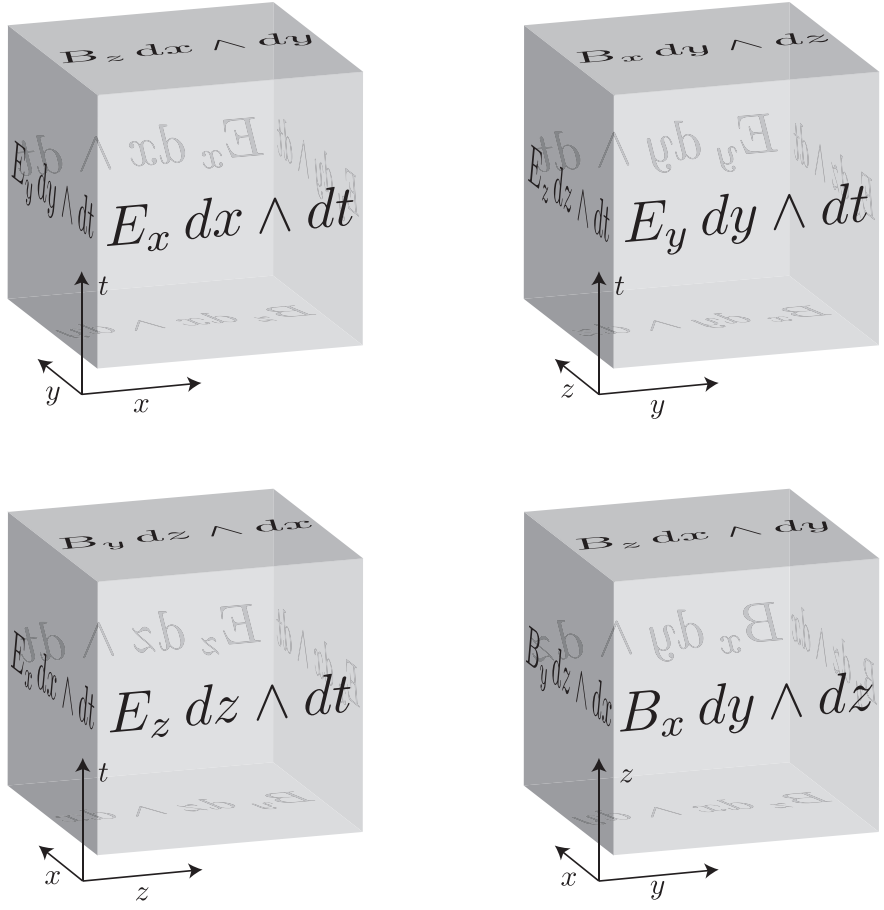

Figure 4: Values of $F$ are stored on the primal 2-faces of a 4-D rectangular grid. Shown here are the three mixed space/time 3-cells, and the one purely spatial 3 -cell (lower right).

Setup. Since $F$ is a 2 -form, its values should live on 2 -faces in this grid. Following the continuous expression of $F$

$$
\begin{aligned}
F=E_{x} \mathrm{~d} x \wedge \mathrm{d} t+E_{y} \mathrm{~d} y \wedge \mathrm{d} t+E_{z} \mathrm{~d} z \wedge d t \\
+B_{x} \mathrm{~d} y \wedge \mathrm{d} z+B_{y} \mathrm{~d} z \wedge \mathrm{d} x+B_{z} \mathrm{~d} x \wedge \mathrm{d} y,
\end{aligned}
$$

and due to the tensor product nature of the regular grid, the exact assignment of each 2-face becomes simple: the six components of $F$ correspond precisely to the six types of 2-faces in a 4-D rectangular grid. Simply assign the values $E_{x} \Delta x \Delta t$ to faces parallel to the $x t$-plane, $E_{y} \Delta y \Delta t$ to faces parallel to the $y t$-plane, and $E_{z} \Delta z \Delta t$ to faces parallel to the $z t$-plane. Likewise, assign $B_{x} \Delta y \Delta z$ to faces parallel to the $y z$-plane, $B_{y} \Delta z \Delta x$ to faces parallel to the $x z$-plane, and $B_{z} \Delta x \Delta y$ to faces parallel to the $x y$-plane. This is pictured in Figure 4.

Let us look at these values on the faces of a typical 4-rectangle $\left[x_{k}, x_{k+1}\right] \times$ $\left[y_{l}, y_{l+1}\right] \times\left[z_{m}, z_{m+1}\right] \times\left[t_{n}, t_{n+1}\right]$. To simplify the notation, we can index each value of $F$ by the midpoint of the 2-face on which it lives: for example, $\left.F\right|_{k+\frac{1}{2}, l, m} ^{n+\frac{1}{2}}$ is stored on the face $\left[x_{k}, x_{k+1}\right] \times\left\{y_{l}\right\} \times\left\{z_{m}\right\} \times\left[t_{n}, t_{n+1}\right]$, parallel to the $x t$-plane. 
Hence, the following values are assigned to the corresponding faces:

$$
\begin{gathered}
x t \text {-face }:\left.E_{x}\right|_{k+\frac{1}{2}, l, m} ^{n+\frac{1}{2}} \Delta x \Delta t \\
y t \text {-face }:\left.E_{y}\right|_{k, l+\frac{1}{2}, m} ^{n+\frac{1}{2}} \Delta y \Delta t \\
z t \text {-face }:\left.E_{z}\right|_{k, l, m+\frac{1}{2}} ^{n+\frac{1}{2}} \Delta z \Delta t \\
y z \text {-face }:\left.B_{x}\right|_{k, l+\frac{1}{2}, m+\frac{1}{2}} ^{n} \Delta y \Delta z \\
x z \text {-face }:\left.B_{y}\right|_{k+\frac{1}{2}, l, m+\frac{1}{2}} ^{n} \Delta z \Delta x \\
x y \text {-face }:\left.B_{z}\right|_{k+\frac{1}{2}, l+\frac{1}{2}, m} ^{n} \Delta x \Delta y .
\end{gathered}
$$

We see that a "staggered grid" arises from the fact that $E$ and $B$ naturally live on 2-faces, not at vertices or 4-cells.

Equations of Motion. The discrete equations of motion are, as in the continuous case,

$$
\mathrm{d} F=0, \quad \mathrm{~d} G=\mathscr{J},
$$

where now these equations are interpreted in the sense of DEC. Let us first look at the DEC interpretation of $\mathrm{d} F$. Since $\mathrm{d} F$ is a discrete 3 -form, it takes values on the 3-faces of each 4-rectangle. Its values are as follows:

$$
\begin{aligned}
x y t \text {-face }: & -\left(\left.E_{x}\right|_{k+\frac{1}{2}, l+1, m} ^{n+\frac{1}{2}}-\left.E_{x}\right|_{k+\frac{1}{2}, l, m} ^{n+\frac{1}{2}}\right) \Delta x \Delta t \\
& +\left(\left.E_{y}\right|_{k+\frac{1}{2}} ^{n+1, l+\frac{1}{2}, m}-\left.E_{y}\right|_{k, l+\frac{1}{2}, m} ^{n+\frac{1}{2}}\right) \Delta y \Delta t \\
& +\left(\left.B_{z}\right|_{k+\frac{1}{2}, l+\frac{1}{2}, m} ^{n+1}-\left.B_{z}\right|_{k+\frac{1}{2}, l+\frac{1}{2}, m} ^{n}\right) \Delta x \Delta y \\
x z t \text {-face }: & -\left(\left.E_{x}\right|_{k+\frac{1}{2}, l, m+1} ^{n+\frac{1}{2}}-\left.E_{x}\right|_{k+\frac{1}{2}, l, m} ^{n+\frac{1}{2}}\right) \Delta x \Delta t \\
& +\left(\left.E_{z}\right|_{k+1, l, m+\frac{1}{2}} ^{n+1}-\left.E_{z}\right|_{k, l, m+\frac{1}{2}} ^{n+\frac{1}{2}}\right) \Delta z \Delta t \\
& -\left(\left.B_{y}\right|_{k+\frac{1}{2}, l, m+\frac{1}{2}} ^{n+1}-\left.B_{y}\right|_{k+\frac{1}{2}, l, m+\frac{1}{2}} ^{n+1}\right) \Delta x \Delta z \\
y z t \text {-face }: & -\left(\left.E_{y}\right|_{k, l+\frac{1}{2}, m+1} ^{n+\frac{1}{2}}-\left.E_{y}\right|_{k, l+\frac{1}{2}, m} ^{n+\frac{1}{2}}\right) \Delta y \Delta t \\
& +\left(\left.E_{z}\right|_{k, l+\frac{1}{2}} ^{n+1, m+\frac{1}{2}}-\left.E_{z}\right|_{k, l, m+\frac{1}{2}} ^{n+\frac{1}{2}}\right) \Delta z \Delta t \\
& +\left(\left.B_{x}\right|_{k, l+\frac{1}{2}, m+\frac{1}{2}} ^{n+1}-\left.B_{x}\right|_{k, l+\frac{1}{2}, m+\frac{1}{2}} ^{n+1}\right) \Delta y \Delta z
\end{aligned}
$$




$$
\begin{aligned}
x y z \text {-face } & :\left(\left.B_{x}\right|_{k+1, l+\frac{1}{2}, m+\frac{1}{2}} ^{n}-\left.B_{x}\right|_{k, l+\frac{1}{2}, m+\frac{1}{2}} ^{n}\right) \Delta y \Delta z \\
& +\left(\left.B_{y}\right|_{k+\frac{1}{2}, l+1, m+\frac{1}{2}} ^{n}-\left.B_{y}\right|_{k+\frac{1}{2}, l, m+\frac{1}{2}} ^{n}\right) \Delta x \Delta z \\
& +\left(\left.B_{z}\right|_{k+\frac{1}{2}, l+\frac{1}{2}, m+1} ^{n}-\left.B_{z}\right|_{k+\frac{1}{2}, l+\frac{1}{2}, m} ^{n}\right) \Delta x \Delta y
\end{aligned}
$$

Setting each of these equal to zero, we arrive at the following four equations:

$$
\begin{aligned}
& \frac{\left.B_{x}\right|_{k, l+\frac{1}{2}, m+\frac{1}{2}} ^{n+1}-\left.B_{x}\right|_{k, l+\frac{1}{2}, m+\frac{1}{2}} ^{n}}{\Delta t}= \\
& \frac{\left.E_{y}\right|_{k, l+\frac{1}{2}, m+1} ^{n+\frac{1}{2}}-\left.E_{y}\right|_{k, l+\frac{1}{2}, m} ^{n+\frac{1}{2}}}{\Delta z}-\frac{\left.E_{z}\right|_{k, l+1, m+\frac{1}{2}} ^{n+\frac{1}{2}}-\left.E_{z}\right|_{k, l, m+\frac{1}{2}} ^{n+\frac{1}{2}}}{\Delta y} \\
& \frac{\left.B_{y}\right|_{k+\frac{1}{2}, l, m+\frac{1}{2}} ^{n+1}-\left.B_{y}\right|_{k+\frac{1}{2}, l, m+\frac{1}{2}} ^{n}}{\Delta t}= \\
& \frac{\left.E_{z}\right|_{k+1, l, m+\frac{1}{2}} ^{n+\frac{1}{2}}-\left.E_{z}\right|_{k, l, m+\frac{1}{2}} ^{n+\frac{1}{2}}}{\Delta x}-\frac{\left.E_{x}\right|_{k+\frac{1}{2}, l, m+1} ^{n+\frac{1}{2}}-\left.E_{x}\right|_{k+\frac{1}{2}, l, m} ^{n+\frac{1}{2}}}{\Delta z} \\
& \frac{\left.B_{z}\right|_{k+\frac{1}{2}, l+\frac{1}{2}, m} ^{n+1}-\left.B_{z}\right|_{k+\frac{1}{2}, l+\frac{1}{2}, m} ^{n}}{\Delta t}= \\
& \frac{\left.E_{x}\right|_{k+\frac{1}{2}, l+1, m} ^{n+\frac{1}{2}}-\left.E_{x}\right|_{k+\frac{1}{2}, l, m} ^{n+\frac{1}{2}}}{\Delta y}-\frac{\left.E_{y}\right|_{k+1, l+\frac{1}{2}, m} ^{n+\frac{1}{2}}-\left.E_{y}\right|_{k, l+\frac{1}{2}, m} ^{n+\frac{1}{2}}}{\Delta x}
\end{aligned}
$$

and

$$
\begin{aligned}
& \frac{\left.B_{x}\right|_{k+1, l+\frac{1}{2}, m+\frac{1}{2}} ^{n}-\left.B_{x}\right|_{k, l+\frac{1}{2}, m+\frac{1}{2}} ^{n}}{\Delta x}+\frac{\left.B_{y}\right|_{k+\frac{1}{2}, l+1, m+\frac{1}{2}} ^{n}-\left.B_{y}\right|_{k+\frac{1}{2}, l, m+\frac{1}{2}} ^{n}}{\Delta y} \\
& \quad+\frac{\left.B_{z}\right|_{k+\frac{1}{2}, l+\frac{1}{2}, m+1} ^{n}-\left.B_{z}\right|_{k+\frac{1}{2}, l+\frac{1}{2}, m} ^{n}}{\Delta z}=0 .
\end{aligned}
$$

These equations are the discrete version of the equations

$$
\partial_{t} \mathbf{B}=-\nabla \times \mathbf{E}, \quad \nabla \cdot \mathbf{B}=0 .
$$

Moreover, since $E$ and $B$ are differential forms, this can also be seen as a discretization of the integral version of Maxwell's equations as well! Because DEC satisfies a discrete Stokes' theorem, this automatically preserves the equivalence between the differential and integral formulations of electromagnetism. 
Doing the same with the equation $\mathrm{d} G=\mathscr{J}$, evaluating on dual 3-faces this time, we arrive at four more equations:

$$
\begin{aligned}
& \frac{\left.D_{x}\right|_{k+\frac{1}{2}, l, m} ^{n+\frac{1}{2}}-\left.D_{x}\right|_{k+\frac{1}{2}, l, m} ^{n-\frac{1}{2}}}{\Delta t}= \\
& \quad \frac{\left.H_{z}\right|_{k+\frac{1}{2}, l+\frac{1}{2}, m} ^{n}-\left.H_{z}\right|_{k+\frac{1}{2}, l-\frac{1}{2}, m} ^{n}}{\Delta y}-\frac{\left.H_{y}\right|_{k+\frac{1}{2}, l, m+\frac{1}{2}} ^{n}-\left.H_{y}\right|_{k+\frac{1}{2}, l, m-\frac{1}{2}} ^{n}}{\Delta z}-\left.J_{x}\right|_{k+\frac{1}{2}, l, m} ^{n} \\
& \frac{\left.D_{y}\right|_{k, l+\frac{1}{2}, m} ^{n+\frac{1}{2}}-\left.D_{y}\right|_{k, l+\frac{1}{2}, m} ^{n-\frac{1}{2}}}{\Delta t}= \\
& \frac{\left.H_{x}\right|_{k, l+\frac{1}{2}, m+\frac{1}{2}} ^{n}-\left.H_{x}\right|_{k, l+\frac{1}{2}, m-\frac{1}{2}} ^{n}}{\Delta z}-\frac{\left.H_{z}\right|_{k+\frac{1}{2}, l+\frac{1}{2}, m} ^{n}-\left.H_{z}\right|_{k-\frac{1}{2}, l+\frac{1}{2}, m} ^{n}}{\Delta x}-\left.J_{y}\right|_{k, l+\frac{1}{2}, m} ^{n} \\
& \quad \frac{\left.\left.D_{y}\right|_{k+l, m} ^{n+\frac{1}{2}}\right|_{k+\frac{1}{2}, l, m+\frac{1}{2}} ^{n}-\left.D_{z}\right|_{k, l, m+\frac{1}{2}} ^{n-\frac{1}{2}}}{\Delta t}= \\
& \Delta x
\end{aligned}
$$

and

$$
\begin{gathered}
\frac{\left.D_{x}\right|_{k+\frac{1}{2}, l, m} ^{n+\frac{1}{2}}-\left.D_{x}\right|_{k-\frac{1}{2}, l, m} ^{n+\frac{1}{2}}}{\Delta x}+\frac{\left.D_{y}\right|_{k, l+\frac{1}{2}, m} ^{n+\frac{1}{2}}-\left.D_{y}\right|_{k, l-\frac{1}{2}, m} ^{n+\frac{1}{2}}}{\Delta y} \\
+\frac{\left.D_{z}\right|_{k, l, m+\frac{1}{2}} ^{n+\frac{1}{2}}-\left.D_{z}\right|_{k, l, m-\frac{1}{2}} ^{n+\frac{1}{2}}}{\Delta z}=\left.\rho\right|_{k, l, m} ^{n+\frac{1}{2}} .
\end{gathered}
$$

This results from storing $G$ on the dual grid, as shown in Figure 5 . This set of equations is the discrete version of

$$
\partial_{t} \mathbf{D}=\nabla \times \mathbf{H}-\mathbf{J}, \quad \nabla \cdot \mathbf{D}=\rho .
$$

After eliminating the redundant divergence equations 4.1) and 4.2) (see Section 5.2 for details) and making the substitutions $\mathbf{D}=\epsilon \mathbf{E}, \mathbf{B}=\mu \mathbf{H}$, the remaining equations are precisely the Yee scheme, as formulated in Bondeson et al. 2005. pp. 67-68).

4.2. Unstructured Spatial Mesh with Uniform Time Steps. We now consider the case of an unstructured grid in space, but with uniform steps in time as advocated in, e.g., Bossavit and Kettunen (1999). Suppose that, instead of a rectangular grid for both space and time, we have an arbitrary space discretization on which we would like to take uniform time steps. (For example, we may be given a tetrahedral mesh of the spatial domain.) This mesh contains two distinct 

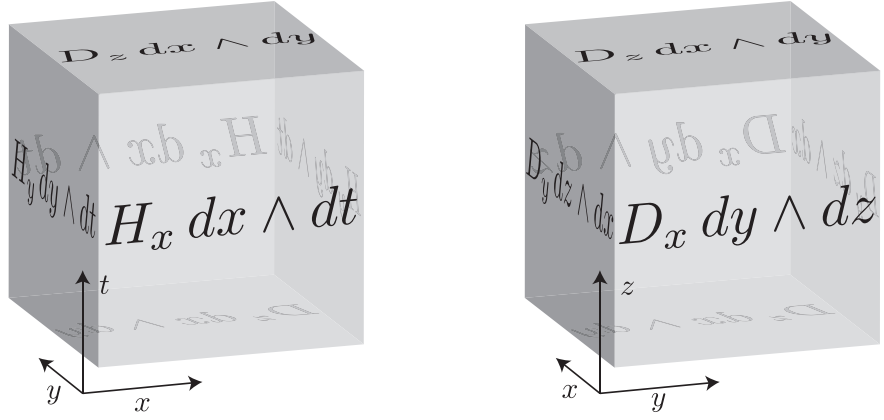

Figure 5: Values of $G=* F$ are stored on dual 2-faces in a rectangular grid. Shown here are a mixed space/time dual 3-cell (left), corresponding to a spacelike primal edge; and a purely spatial dual 3-cell (right), corresponding to a timelike primal edge. There are also two other mixed space/time cells, as in Figure 4, that are not shown here.

types of 2-faces. First, there are triangular faces that live entirely in the space mesh at a single position in time. Every edge of such a face is spacelike-that is, it has positive length—so the causality operator defined in Section 3.2 takes the value $\kappa=1$. Second, there are rectangular faces that live between time steps. These faces consist of a single spacelike edge extruded by one time step. Because they have one timelike edge, these faces satisfy $\kappa=-1$. Again, the circumcentricdual DEC framework applies directly to this type of mesh, since the prismal extrusion of a 3-simplex still has a circumcenter.

Setup. Again, we can characterize the discrete values of $F$ by looking at the continuous expression

$$
F=E \wedge \mathrm{d} t+B
$$

Therefore, let us assign $B$ to the purely spacelike faces and $E \Delta t$ to the mixed space/time faces. Looking at $G=* F$ shows that mixed dual faces should store $H \Delta t$ and spacelike dual faces should store $D$; see Figure 6 .

Equations of Motion. As in Bossavit (1998), we can store the values of each differential form over every spatial element in an array, using the method described in Section 3.2. This leads to the arrays $B^{n}$ and $H^{n}$ at whole time steps $n$, and $E^{n+1 / 2}$ and $D^{n+1 / 2}$ at half time steps. Let $\mathrm{d}_{1}$ denote the edges-to-faces incidence matrix for the spatial domain. That is, $d_{1}$ is the matrix corresponding to the discrete exterior derivative, taken only in space, from primal 1-forms to primal 2-forms. Similarly, the transpose $d_{1}^{T}$ corresponds to the exterior derivative from spatial dual 1 -forms to dual 2-forms. Then the equation $\mathrm{d} F=0$, evaluated on all prismal 

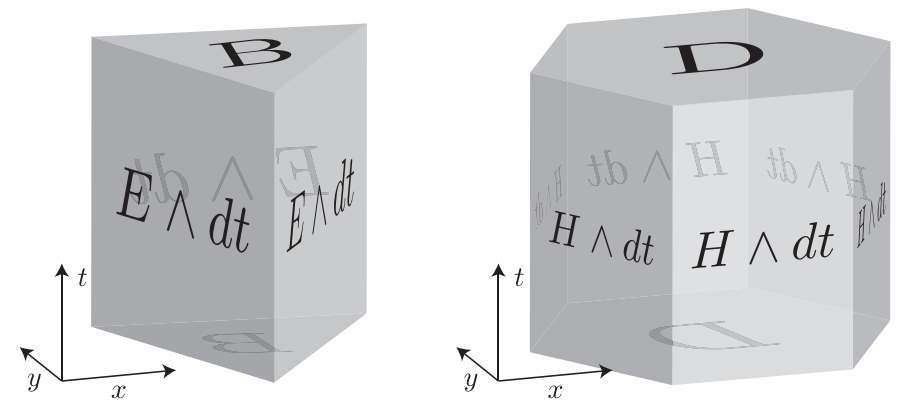

Figure 6: For an unstructured spatial mesh, $F$ is stored on primal 2-faces (left), while $G=* F$ is stored on dual 2-faces (right). Shown here are the values on mixed space/time 3-cells. (The purely spatial 3-cells, which correspond to the divergence equations and do not contribute to the equations of motion, are not shown.)

3-faces becomes

$$
\frac{B^{n+1}-B^{n}}{\Delta t}=-\mathrm{d}_{1} E^{n+1 / 2}
$$

Likewise, the equation $\mathrm{d} G=\mathscr{J}$, evaluated on all space/time 3 -faces in the dual mesh, becomes

$$
\frac{D^{n+1 / 2}-D^{n-1 / 2}}{\Delta t}=\mathrm{d}_{1}^{T} H^{n}-J^{n} .
$$

We can also evaluate $\mathrm{d} F=0$ and $\mathrm{d} G=\mathscr{J}$ on spacelike 3-faces, e.g, tetrahedra; these simply yield the discrete versions of the divergence conditions for $B$ and $D$, which can be eliminated.

Therefore, the DEC scheme for such a mesh is equivalent to Bossavit and Kettunen's Yee-like scheme; additionally, when the spatial mesh is taken to be rectangular, this integrator reduces to the standard Yee scheme. However, we now have solid foundations to extend this integrator to handle asynchronous updates for improved efficiency.

4.3. Unstructured Spatial Mesh with Asynchronous Time Steps. Instead of picking the same time step size for every element of the spatial mesh, as in the previous two sections, it is often more efficient to assign each element its own, optimized time step, as done in Lew et al. 2003) for problems in elastodynamics. In this case, rather than the entire mesh evolving forward in time simultaneously, individual elements advance one-by-one, asynchronously-hence the name asynchronous variational integrator (AVI). As we will prove in Section 5 this asynchronous update process will maintain the variational nature of the integration scheme. Here, we again allow the spatial mesh to be unstructured. 

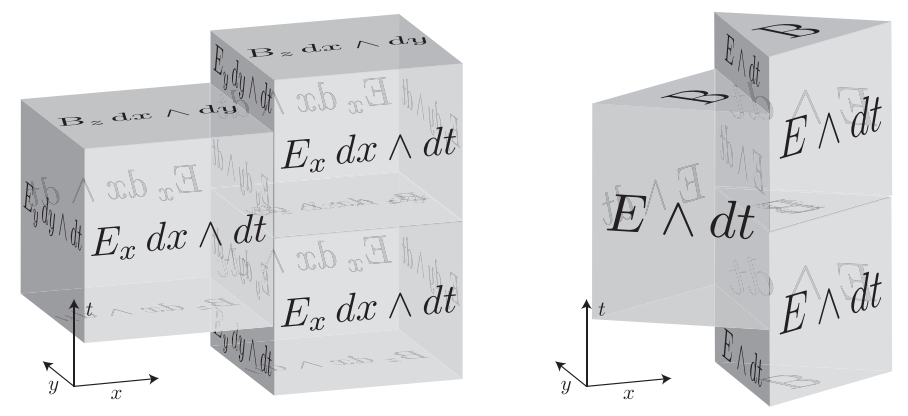

Figure 7: Shown here is part of an AVI mesh, for a rectangular spatial mesh (left) and for an unstructured spatial mesh (right). The different heights of the spacetime prisms reflect the fact that elements can take different time steps from one another. Moreover, these time steps can be asynchronous, as seen in the mismatch between the horizontal faces.

Setup. After choosing a primal space mesh, assign each spatial 2-face (e.g., triangle) $\sigma$ its own discrete time set

$$
\Theta_{\sigma}=\left\{t_{\sigma}^{0}<\cdots<t_{\sigma}^{N_{\sigma}}\right\} .
$$

For example, one might assign each face a fixed time step size $\Delta t_{\sigma}=t_{\sigma}^{n+1}-t_{\sigma}^{n}$, taking equal time steps within each element, but with $\Delta t$ varying across elements. We further require for simplicity of explanation that, except for the initial time, no two faces take the same time step: that is, $\Theta_{\sigma} \cap \Theta_{\sigma^{\prime}}=\left\{t_{0}\right\}$ for $\sigma \neq \sigma^{\prime}$.

In order to keep proper time at the edges $e$ where multiple faces with different time sets meet, we let

$$
\Theta_{e}=\bigcup_{\sigma \ni e} \Theta_{\sigma}=\left\{t_{e}^{0} \leq \cdots \leq t_{e}^{N_{e}}\right\} .
$$

Therefore the mixed space-time 2-faces, which correspond to the edge $e$ extruded over a time step, are assigned the set of intermediate times

$$
\Theta_{e}^{\prime}=\left\{t_{e}^{1 / 2} \leq \cdots \leq t_{e}^{N_{e}-1 / 2}\right\}
$$

where $t_{e}^{k+1 / 2}=\left(t_{e}^{k+1}+t_{e}^{k}\right) / 2$. The values stored on a primal AVI mesh are shown in Figure 7 .

Since $\Theta_{e} \supset \Theta_{\sigma}$ when $e \subset \sigma$, each spatial edge $e$ takes more time steps than any one of its incident faces $\sigma$; as a result, it is not possible in general to construct a circumcentric dual on the entire spacetime AVI mesh, since the mesh is not prismal and hence the circumcenter may not exist. Instead, we find the circumcentric dual to the spatial mesh, and assign same time steps to the primal and 
dual elements

$$
\Theta_{* \sigma}=\Theta_{\sigma}, \quad \Theta_{* e}=\Theta_{e} .
$$

This results in well-defined primal and dual cells for each 2-element in spacetime, and hence a Hodge star for this order. (A Hodge star on forms of different order is not needed to formulate Maxwell's equations.)

Equations of Motion. The equation $\mathrm{d} F=0$, evaluated on a mixed space/time 3-cell, becomes

$$
\frac{B_{\sigma}^{n+1}-B_{\sigma}^{n}}{t_{\sigma}^{n+1}-t_{\sigma}^{n}}=-\mathrm{d}_{1} \sum\left\{E_{e}^{m+1 / 2}: t_{\sigma}^{n}<t_{e}^{m+1 / 2}<t_{\sigma}^{n+1}\right\} .
$$

Similarly, the equation $\mathrm{d} G=\mathscr{J}$ becomes

$$
\frac{D_{e}^{m+1 / 2}-D_{e}^{m-1 / 2}}{t_{e}^{m+1 / 2}-t_{e}^{m-1 / 2}}=\mathrm{d}_{1}^{T}\left(H_{\sigma}^{n} \nVdash_{\left\{t_{\sigma}^{n}=t_{e}^{m}\right\}}\right)-J_{e}^{m},
$$

where $\nVdash_{\left\{t_{\sigma}^{n}=t_{e}^{m}\right\}}$ equals 1 when face $\sigma$ has $t_{\sigma}^{n}=t_{e}^{m}$ for some $n$, and 0 otherwise. (That is, the indicator function "picks out" the incident face that lives at the same time step as this edge.)

Solving an initial value problem can then be summarized by the following update loop:

(1) Pick the minimum time $t_{\sigma}^{n+1}$ where $B_{\sigma}^{n+1}$ has not yet been computed.

(2) Advance $B_{\sigma}^{n+1}$ according to Equation 4.3 .

(3) Update $H_{\sigma}^{n+1}=*_{\mu}^{-1} B_{\sigma}^{n+1}$.

(4) Advance $D_{e}^{m+3 / 2}$ on neighboring edges $e \subset \sigma$ according to Equation 4.4.

(5) Update $E_{e}^{m+3 / 2}=*_{\epsilon}^{-1} D_{e}^{m+3 / 2}$.

Iterative Time Stepping Scheme. As detailed in Lew et al. (2003) for elastodynamics, the explicit AVI update scheme can be implemented by selecting mesh elements from a priority queue, sorted by time, and iterating forward. However, as written above, the scheme is not strictly iterative, since Equation 4.4 depends on past values of $E$. This can be easily fixed by rewriting the AVI scheme to advance in the variables $A$ and $E$ instead, where the potential $A$ effectively stores the cumulative contribution of $E$ to the value of $B$ on neighboring faces. Compared to the AVI for elasticity, $A$ plays the role of the positions $\mathbf{x}$, while $E$ plays the role of the (negative) velocities $\dot{\mathbf{x}}$. The algorithm is given as pseudocode in Figure 8 . Note that if all elements take uniform time steps, the AVI reduces to the Bossavit-Kettunen scheme.

Numerical Experiments. We first present a simple numerical example demonstrating the good energy behavior of our asynchronous integrator. The AVI was used to integrate in time over a 2-D rectangular cavity with perfectly electrically 


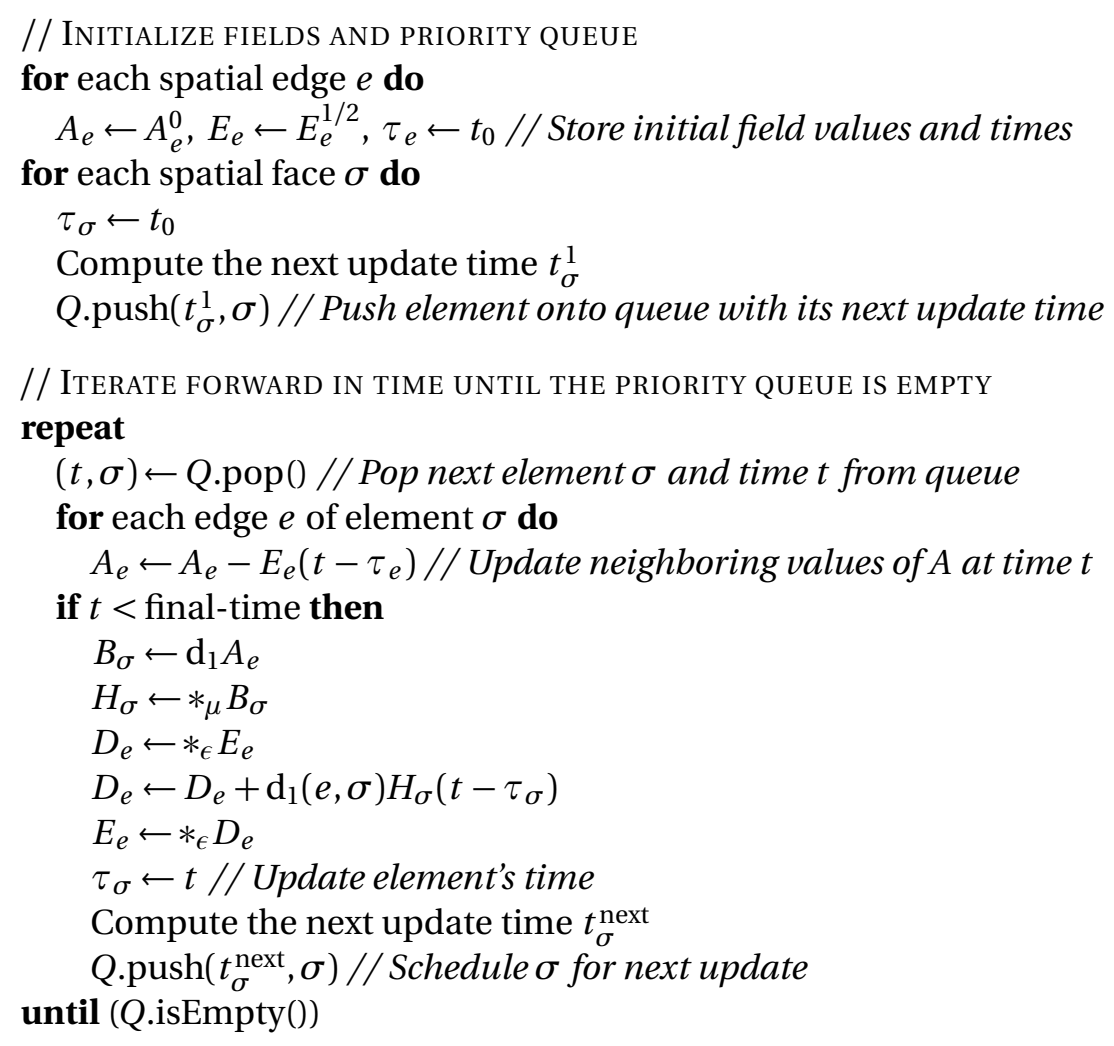

Figure 8: Pseudocode for our Asynchronous Variational Integrator, implemented using a priority queue data structure for storing and selecting the elements to be updated.

conducting (PEC) boundaries, so that $E$ vanishes at the boundary of the domain. $E$ was given random values at the initial time, so as to excite all frequency modes, and integrated for 8 seconds. Each spatial element was given a time step equal to $1 / 10$ of the stability-limiting time step determined by the CFL condition.

This simulation was done for two different spatial discretizations. The first is a uniform discretization so that each element has identical time step size, which coincides exactly with the Yee scheme. The second discretization randomly partitioned the $x$ - and $y$-axes, so that each element has completely unique spatial dimensions and time step size, and so the update rule is truly asynchronous. The energy plot for the uniform Yee discretization is shown in Figure 9. while the energy for the random discretization is shown in Figure 10. Even for a completely random, irregular mesh, our asynchronous integrator displays near-energy preservation qualities. Such numerical behavior stems from the variational nature of our integrator, which will be detailed in Section 5 . 


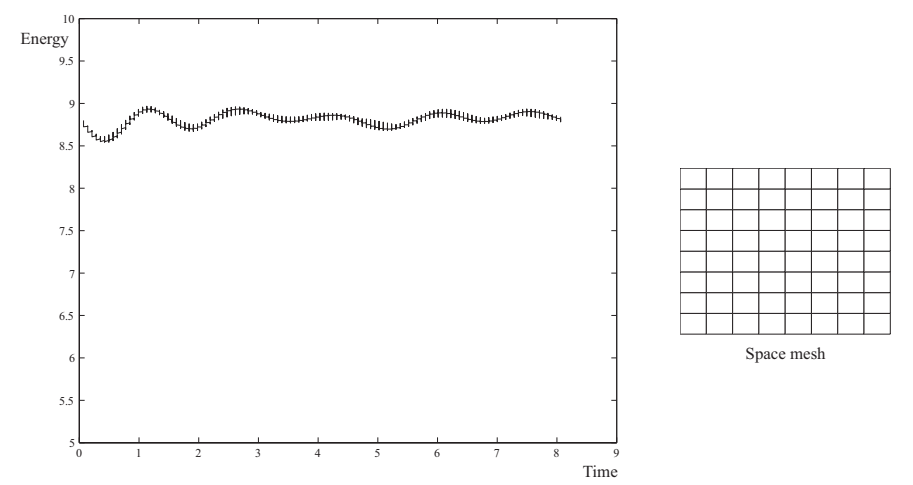

Figure 9: Energy vs. time for the AVI with uniform space and time discretization. This is the special case where the AVI reproduces the Yee scheme - which is well known to have good energy conservation properties, as seen here. (The vertical "tick marks" on the plot show where the elements become synchronized, since they take uniform time steps.)

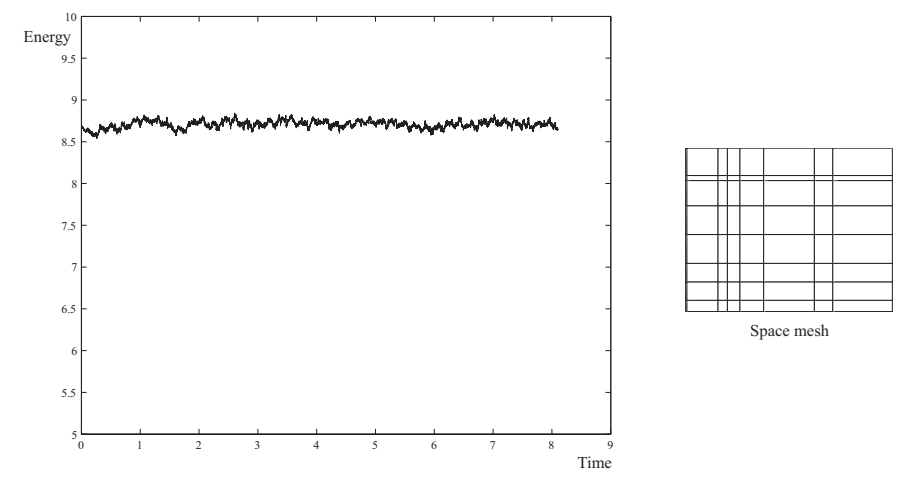

Figure 10: Energy vs. time for the AVI with random spatial discretization and fully asynchronous time steps. Despite the lack of regularity in the mesh and time steps, the AVI maintains the good energy behavior displayed by the Yee scheme.

In addition, we tested the performance of the AVI method with regard to computing the resonant frequencies of a 3-D rectangular cavity, but using an unstructured tetrahedral spatial mesh. While the resonant frequencies are relatively simple to compute analytically, nodal finite element methods are well known to produce spurious modes for this type of simulation. By contrast, as shown in Fig. ure 11, the AVI simulation produces a resonance spectrum in close agreement with theory. Furthermore, by refining the mesh close to the spatial boundary, while using a coarser discretization in the interior, we were able to achieve these results with less computational effort than a uniformly fine mesh would require, 

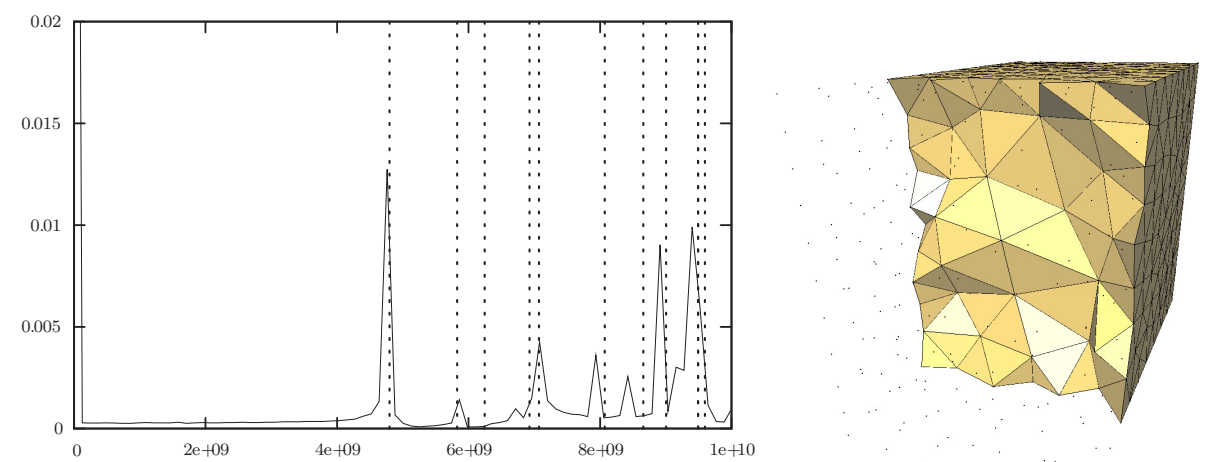

Figure 11: To produce the power spectrum shown at left, the electric field $E$ was initialized with random data (to excite all frequencies) and integrated forward in time, measuring the field strength at a particular sample point for every time step, and then performing a discrete Fourier transform. The locations of the amplitude "spikes" are in close agreement with the analytic resonant frequencies, shown by the dashed vertical lines. The spatial mesh, shown at right, was refined closer to the boundary, and coarser in the interior, allowing the AVI to produce this result with fewer total steps than uniform-time-stepping would require.

since the time steps were selected to be proportional to the respective element sizes.

4.4. Fully Unstructured Spacetime Mesh. Finally, we look at the most general possible case: an arbitrary discretization of spacetime, such as a simplicial 4complex. Such a mesh is completely relativistically covariant, so that $F$ cannot be objectively separated into the components $E$ and $B$ without a coordinate frame. In most engineering applications, relativistic effects are insignificant, so a $3+1$ mesh (as in the previous subsections) is almost always adequate, and avoids the additional complications of spacetime mesh construction. Still, we expect that there are scientific applications where a covariant discretization of electromagnetism may be very useful. For example, many implementations of numerical general relativity (using Regge calculus for instance) are formulated on simplicial 4-complexes; one might wish to simulate the interaction of gravity with the electromagnetic field, or charged matter, on such a mesh.

Spacetime Mesh Construction. First, a quick caution on mesh construction: since the Lorentz metric is not positive definite, it is possible to create edges that have length 0 , despite connecting two distinct points in $\mathbb{R}^{3,1}$ (so-called "null" or "lightlike" edges). Meshes containing such edges are degenerate-akin to 
a Euclidean mesh containing a triangle with two identical points. In particular, the DEC Hodge star is undefined for 0 -volume elements (due to division by zero). Even without 0 -volume elements, it is still possible for a spacetime mesh to violate causality, so extra care should be taken. Methods to construct causality-respecting spacetime meshes over a given spatial domain can be found in, e.g., Erickson, Guoy, Sullivan, and Üngör (2005) and Thite (2005).

When the mesh contains no inherent choice of a time direction, there is no canonical way to split $F$ into $E$ and $B$. Therefore, one must set up the problem by assigning values of $F$ directly to 2-cells (or equivalently, assigning values of $A$ to 1-cells). For initial boundary value problems, one might choose to have the initial and final time steps be prismal, so that $E$ and $B$ can be used for initial and final values, while the internal discretization is general.

Equations of Motion. The equations $\mathrm{d} F=0$ and $\mathrm{d} G=\mathscr{J}$ can be implemented directly in DEC. Since this mesh is generally unstructured, there is no simple algorithm as the ones we presented above. Instead, the equations on $F$ results in a sparse linear system which, given proper boundary conditions, can be solved globally with direct or iterative solvers. However, it is clear that the previous three examples that the methods of Yee, Bossavit-Kettunen, and our AVI integrator are special cases where the global solution is particularly simple to compute via synchronous or asynchronous time updates.

Mesh Construction and Energy Behavior. It is known that, while variational integrators in mechanics do not preserve energy exactly, they have excellent energy behavior, in that it tends to oscillate close to the exact value. This is only true, however, when the integrator takes time steps of uniform size; adaptive and other non-uniform stepping approaches can give poor results unless additional measures are taken to enforce good energy behavior. (See Hairer et al., 2006. Chapter VIII for a good discussion of this problem for mechanics applications.)

Therefore, there is no reason to expect that arbitrary meshes of spacetime will yield energy results as good as the Yee, Bossavit-Kettunen, and AVI schemes. However, if one is taking a truly covariant approach to spacetime, "energy" is not even defined without specifying a time coordinate. Likewise, one would not necessarily expect good energy behavior from the other methods with respect to an arbitrary transformation of spatial coordinates. Which sort of mesh to choose is thus highly application-dependent.

\section{Theoretical REsults}

In this section, we complete our exposition with a number of theoretical results about the discrete and continuous Maxwell's equations. In particular, we show 
that the DEC formulation of electromagnetism derives from a discrete Lagrangian variational principle, and that this formulation is consequently multisymplectic. Furthermore, we explore the gauge symmetry of Maxwell's equations, and detail how a particular choice of gauge eliminates the equation for $\nabla \cdot \mathbf{D}-\rho$ from the Euler-Lagrange equations, while preserving it automatically as a momentum map.

Theorem 5.1. The discrete Maxwell's equations are variational.

Proof. The idea of this proof is to emulate the derivation of the continuous Maxwell's equations from Section 2. Interpreting this in the sense of DEC, we will obtain the discrete Maxwell's equations.

Given a discrete 1-form $A$ and dual source 3-form $\mathscr{J}$, define the discrete Lagrangian 4-form

$$
\mathscr{L}_{d}=-\frac{1}{2} \mathrm{~d} A \wedge * \mathrm{~d} A+A \wedge \mathscr{J},
$$

with the corresponding discrete action principle

$$
S_{d}[A]=\left\langle\mathscr{L}_{d}, K\right\rangle .
$$

Then, taking a discrete 1-form variation $\alpha$ vanishing on the boundary, the corresponding variation of the action is

$$
\mathbf{d} S_{d}[A] \cdot \alpha=\langle-\mathrm{d} \alpha \wedge * \mathrm{~d} A+\alpha \wedge \mathscr{J}, K\rangle=\langle\alpha \wedge(-\mathrm{d} * \mathrm{~d} A+\mathscr{J}), K\rangle .
$$

(Here we use the bold $\mathbf{d}$ to indicate that we are differentiating over the smooth space of discrete forms $A$, as opposed to differentiating over discrete spacetime, for which we use d.) Setting this equal to 0 for all variations $\alpha$, the resulting discrete Euler-Lagrange equations are therefore $\mathrm{d} * \mathrm{~d} A=\mathscr{J}$. Defining the discrete 2 -forms $F=\mathrm{d} A$ and $G=* F$, this implies $\mathrm{d} F=0$ and $\mathrm{d} G=\mathscr{J}$, the discrete Maxwell's equations.

5.1. Multisymplecticity. The concept of multisymplecticity for Lagrangian field theories was developed in Marsden et al. (1998), where it was shown to arise from the boundary terms for general variations of the action, i.e., those not restricted to vanish at the boundary. As originally presented, the Cartan form $\theta_{\mathscr{L}}$ is an $(n+1)$-form, where the $n$-dimensional boundary integral is then obtained by contracting $\theta_{\mathscr{L}}$ with a variation. The multisymplectic $(n+2)$-form $\omega_{\mathscr{L}}$ is then given by $\omega_{\mathscr{L}}=-\mathbf{d} \theta_{\mathscr{L}}$. Contracting $\omega_{\mathscr{L}}$ with two arbitrary variations gives an $n$-form that vanishes when integrated over the boundary, a result called the multisymplectic form formula, which results from the identity $\mathbf{d}^{2}=0$. In the special case of mechanics, where $n=0$, the boundary consists of the initial and final time points; hence, this implies the usual result that the symplectic 2-form $\omega_{L}$ is preserved by the time flow. 
Alternatively, as communicated to us by Patrick (2004), one can view the Cartan form $\theta_{\mathscr{L}}$ as an $n$-form-valued 1-form, and the multisymplectic form $\omega_{\mathscr{L}}$ as an $n$-form-valued 2-form. Therefore, one simply evaluates these forms on tangent variations to obtain a boundary integral, rather than taking contractions. These two formulations are equivalent on smooth spaces. However, we will adopt Patrick's latter definition, since it is more easily adapted to problems on discrete meshes: $\theta_{\mathscr{L}}$ and $\omega_{\mathscr{L}}$ remain smooth 1- and 2-forms, respectively, but their $n$ form values are now taken to be discrete. See Figure 12 for an illustration of the discrete multisymplectic form formula.

Theorem 5.2. The discrete Maxwell's equations are multisymplectic.

Proof. Let $K \subset \mathscr{K}$ be an arbitrary subcomplex, and consider the discrete action functional $S_{d}$ restricted to $K$. Suppose now that we take a discrete variation $\alpha$, without requiring it to vanish on the boundary $\partial K$. Then variations of the action contain an additional boundary term

$$
\mathbf{d} S_{d}[A] \cdot \alpha=\langle\alpha \wedge(-\mathrm{d} * \mathrm{~d} A+\mathscr{J}), K\rangle+\langle\alpha \wedge * d A, \partial K\rangle .
$$

Restricting to the space of potentials $A$ that satisfy the discrete Euler-Lagrange equations, the first term vanishes, leaving only

$$
\mathbf{d} S_{d}(A) \cdot \alpha=\langle\alpha \wedge * \mathrm{~d} A, \partial K\rangle
$$

Then we can define the Cartan form $\theta_{\mathscr{L}_{d}}$ by

$$
\theta_{\mathscr{L}_{d}} \cdot \alpha=\alpha \wedge * \mathrm{~d} A .
$$

Since $\theta_{\mathscr{L}_{d}}$ takes a tangent vector $\alpha$ and produces a discrete 3 -form on the boundary of the subcomplex, it is a smooth 1-form taking discrete 3 -form values. Now, since the space of discrete forms is itself actually continuous, we can take the exterior derivative in the smooth sense on both sides of Equation 5.1. Evaluating along another first variation $\beta$ (again restricted to the space of Euler-Lagrange solutions), we then get

$$
\mathbf{d}^{2} S_{d}[A] \cdot \alpha \cdot \beta=\langle\mathbf{d} \theta \cdot \alpha \cdot \beta, \partial K\rangle .
$$

Finally, defining the multisymplectic form $\omega_{\mathscr{L}_{d}}=-\mathbf{d} \theta_{\mathscr{L}_{d}}$, and using the fact that $\mathbf{d}^{2} S_{d}=0$, we get the relation

$$
\left\langle\omega_{\mathscr{L}_{d}} \cdot \alpha \cdot \beta, \partial K\right\rangle=0
$$

for all variations $\alpha, \beta$; Equation 5.2 is a discrete version of the multisymplectic form formula. Since this holds for any subcomplex $K$, it follows that these schemes are multisymplectic. 


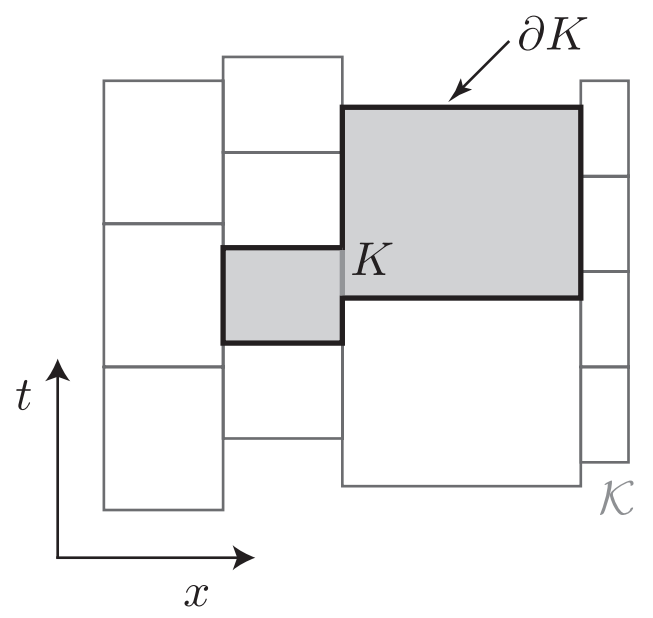

Figure 12: To illustrate the discrete multisymplectic form formula [5.2), we have here a 2-D asynchronous-time mesh $\mathscr{K}$, where the shaded region is an arbitrary subcomplex $K \subset \mathscr{K}$. Given any two variations $\alpha, \beta$ of the field, and the multisymplectic form $\omega_{\mathscr{L}_{d}}$, the formula states that $\omega_{\mathscr{L}_{d}} \cdot \alpha \cdot \beta$ vanishes when integrated over the boundary $\partial K$ (shown in bold).

5.2. Gauge Symmetry Reduction and Covariant Momentum Maps. We now explore the symmetry of Maxwell's equations under gauge transformations. This symmetry allows us to reduce the equations by eliminating the time component of $A$ (for some chosen time coordinate), effectively fixing the electric scalar potential to zero. Because this is an incomplete gauge, there is a remaining gauge symmetry, and hence a conserved momentum map. This conserved quantity turns out to be the charge density $\rho=\nabla \cdot \mathbf{D}$, which justifies its elimination from the Euler-Lagrange equations. These calculations are done with differential forms and exterior calculus, hence they apply equally to the smooth and discrete cases of electromagnetism.

5.3. Choosing a Gauge. Because Maxwell's equations only depend on $\mathrm{d} A$, they are invariant under gauge transformations of the form $A \mapsto A+\mathrm{d} f$, where $f$ is any scalar function on spacetime. If we fix a time coordinate, we can now choose the Weyl gauge, so that the time component $A_{t}=0$. Therefore, we can assume that

$$
A=A_{x} \mathrm{~d} x+A_{y} \mathrm{~d} y+A_{z} \mathrm{~d} z .
$$

In fact, $A_{x}, A_{y}, A_{z}$ are precisely the components of the familiar vector potential $\mathbf{A}$, i.e., $A=\mathbf{A}^{b}$.

5.4. Reducing the Equations. Having fixed the gauge and chosen a time coordinate, we can now define two new "partial exterior derivative" operators, $\mathrm{d}_{t}$ (time) 
and $\mathrm{d}_{s}$ (space), where $\mathrm{d}=\mathrm{d}_{t}+\mathrm{d}_{s}$. Since $A$ contains no $\mathrm{d} t$ terms, $\mathrm{d}_{s} A$ is a 2 -form containing only the space terms of $\mathrm{d} A$, while $\mathrm{d}_{t} A$ contains the terms involving both space and time. That is,

$$
\mathrm{d}_{t} A=E \wedge \mathrm{d} t, \quad \mathrm{~d}_{s} A=B .
$$

Restricted to this subspace of potentials, the Lagrangian density then becomes

$$
\begin{aligned}
\mathscr{L} & =-\frac{1}{2}\left(\mathrm{~d}_{t} A+\mathrm{d}_{s} A\right) \wedge *\left(\mathrm{~d}_{t} A+\mathrm{d}_{s} A\right)+A \wedge \mathscr{J} \\
& =-\frac{1}{2}\left(\mathrm{~d}_{t} A \wedge * \mathrm{~d}_{t} A+\mathrm{d}_{s} A \wedge * \mathrm{~d}_{s} A\right)+A \wedge J \wedge \mathrm{d} t
\end{aligned}
$$

Next, varying the action along a restricted variation $\alpha$ that vanishes on $\partial X$,

$$
\begin{aligned}
\mathbf{d} S[A] \cdot \alpha & =\int_{X}\left(\mathrm{~d}_{t} \alpha \wedge D-\mathrm{d}_{s} \alpha \wedge H \wedge \mathrm{d} t+\alpha \wedge J \wedge \mathrm{d} t\right) \\
& =\int_{X} \alpha \wedge\left(\mathrm{d}_{t} D-\mathrm{d}_{s} H \wedge \mathrm{d} t+J \wedge \mathrm{d} t\right) .
\end{aligned}
$$

Setting this equal to zero by Hamilton's principle, one immediately gets Ampère's law as the sole Euler-Lagrange equation. The divergence constraint $\mathrm{d}_{s} D=\rho$, corresponding to Gauss' law, has been eliminated via the restriction to the Weyl gauge.

Noether's Theorem Implies Automatic Preservation of Gauss' Law. Let us restrict $A$ to be an Euler-Lagrange solution in the Weyl gauge, but remove the previous requirement that variations $\alpha$ be fixed at the initial time $t_{0}$ and final time $t_{f}$. Then, varying the action along this new $\alpha$, the Euler-Lagrange term disappears, but we now pick up an additional boundary term due to integration by parts

$$
\mathbf{d} S[A] \cdot \alpha=\left.\int_{\Sigma} \alpha \wedge D\right|_{t_{0}} ^{t_{f}}
$$

where $\Sigma$ denotes a Cauchy surface of $X$, corresponding to the spatial domain. If we vary along a gauge transformation $\alpha=\mathrm{d}_{s} f$, then this becomes

$$
\mathbf{d} S[A] \cdot \mathrm{d}_{s} f=\left.\int_{\Sigma} \mathrm{d}_{s} f \wedge D\right|_{t_{0}} ^{t_{f}}=-\left.\int_{\Sigma} f \wedge \mathrm{d}_{s} D\right|_{t_{0}} ^{t_{f}}
$$

Alternatively, plugging $\alpha=\mathrm{d}_{s} f$ into Equation 5.3, we get

$$
\mathbf{d} S[A] \cdot \mathrm{d}_{s} f=\int_{X} \mathrm{~d}_{s} f \wedge J \wedge \mathrm{d} t=-\int_{X} f \wedge \mathrm{d}_{s} J \wedge \mathrm{d} t=-\int_{X} f \wedge \mathrm{d}_{t} \rho=-\int_{\Sigma} f \wedge \rho||_{t_{0}}^{t_{f}} .
$$


Since these two expressions are equal, and $f$ is an arbitrary function, it follows that

$$
\left.\left(\mathrm{d}_{s} D-\rho\right)\right|_{t_{0}} ^{t_{f}}=0 .
$$

This indicates that $\mathrm{d}_{s} D-\rho$ is a conserved quantity, a momentum map, so if Gauss' law holds at the initial time, then it holds for all subsequent times as well.

5.5. Boundary Conditions and Variational Structure. It should be noted that the variational structure and symmetry of Maxwell's equations may be affected by the boundary conditions that one chooses to impose. There are many boundary conditions that one can specify independent of the initial values, such as the PEC condition used in the numerical example in Section 4.3 . However, one can imagine more complicated boundary conditions where which the boundary interacts nontrivially with the interior of the domain - such as dissipative or forced boundary conditions, where energy/momentum is removed from or added to the system. In these cases, one will obviously not conclude that the charge density $\nabla \cdot \mathbf{D}$ is conserved, but more generally that the change in charge is related to the flux through the spatial boundary. This is because, in the momentum map derivation above, the values of $f$ on the initial time slice causally affects its values on the spatial boundary at intermediate times, not just on the final time slice. Thus, the spatial part of $\partial X$ cannot be neglected for arbitrary boundary conditions.

\section{Conclusion}

The continued success of the Yee scheme for many applications of computational electromagnetism, for over four decades, illustrates the value of structurepreserving numerical integrators for Maxwell's equations. Recent advances by, among others, Bossavit and Kettunen, and Gross and Kotiuga, have demonstrated the important role of compatible spatial discretization using differential forms, allowing for Yee-like schemes that apply on generalized spatial meshes. In this paper, we have extended this approach by considering discrete forms on spacetime, encapsulating both space and time discretization, and have derived a general family of geometric numerical integrators for Maxwell's equations. Furthermore, since we have derived these integrators from a discrete variational principle, the resulting methods are provably multisymplectic and momentummap-preserving, and they experimentally show correct global energy behavior. Besides proving the variational nature of well-known techniques such as the Yee and Bossavit-Kettunen schemes, we have also introduced a new asynchronous integrator, so that time step sizes can be taken non-uniformly over the spatial 
domain for increased efficiency, while still maintaining the desirable variational and energy behavior of the other methods.

Future Work. One promising avenue for future work involves increasing the order of accuracy of these methods by deriving higher-order discrete Hodge star operators. While this would involve redefining the Hodge star matrix to be nondiagonal, the discrete Maxwell's equations would remain formally the same, and hence there would be no change in the variational or multisymplectic properties proven here. We are currently exploring the development of a spectrally accurate spatial Hodge star, which might make these geometric schemes competitive for applications where non-variational spectral codes are currently favored.

Additionally, the recent work of Kale and Lew (2007) has shown that AVIs can be implemented as parallel algorithms for solid mechanics simulations. This uses the fact that, due to the asynchronous update procedure, an element does not need information from every one of its neighbors at every time step, which lessens the need for communication among parallel nodes. The resulting parallel AVIs, or PAVIs, can therefore take advantage of parallel computing architecture for improved efficiency. It is reasonable to expect that the same might be done in the case of our electromagnetic AVI.

While we have experimentally observed the fact that variational integrators exhibit near-energy conservation, little is known about this behavior from a theoretical standpoint. In the case of ODEs in mechanics, backwards error analysis has shown that these methods exactly integrate a nearby smooth Hamiltonian system, although not much known about how this relates to the discrete variational principle on the Lagrangian side. Some initial work has been done in Oliver, West, and Wulff (2004) to understand, also by a backward error analysis approach, why discrete multisymplectic methods also display good energy behavior.

Finally, variational methods using discrete spacetime forms may be developed for field theories other than electromagnetism. Promising candidates include numerical general relativity and fluid dynamics, although the latter is complicated by the difficulty in finding a proper discretization of the infinite-dimensional diffeomorphism group. If discrete Lagrangian densities are developed for these theories, it should be straightforward to combine them with the electromagnetic Lagrangian, resulting in numerical methods to simulate, e.g., gravity coupled with an electromagnetic field, or the dynamics of a charged or magnetic fluid.

\section{ACKNOWLEDGMEnTs}

We would like to thank several people for their inspiration and suggestions. First of all, Alain Bossavit for suggesting many years ago that we take the present 
DEC approach to computational electromagnetism, and for his excellent lectures at Caltech on the subject. Second, Michael Ortiz and Eva Kanso for their ongoing interactions on related topics and suggestions. We also thank Doug Arnold, Uri Ascher, Robert Kotiuga, Melvin Leok, Adrian Lew, and Matt West for their feedback and encouragement. In addition, the 3-D AVI simulations shown in Figure 11 were programmed and implemented by Patrick Xia, as part of a Summer Undergraduate Research Fellowship at Caltech.

\section{REFERENCES}

Arnold, D. N., R. S. Falk, and R. Winther (2006), Finite element exterior calculus, homological techniques, and applications. Acta Numer., 15, 1-155.

Auchmann, B., and S. Kurz (2006), A geometrically defined discrete Hodge operator on simplicial cells. IEEE Trans. Magn., 42 (4), 643-646.

Bondeson, A., T. Rylander, and P. Ingelström (2005), Computational electromagnetics, volume 51 of Texts in Applied Mathematics. Springer, New York.

Bossavit, A. (1998), Computational electromagnetism. Electromagnetism, Academic Press Inc., San Diego, CA. Variational formulations, complementarity, edge elements.

Bossavit, A., and L. Kettunen (1999), Yee-like schemes on a tetrahedral mesh, with diagonal lumping. Int. J. Numer. Modell., 12 (1-2), 129-142.

Bossavit, A., and L. Kettunen (2000), Yee-like schemes on staggered cellular grids: A synthesis between FIT and FEM approaches. IEEE Trans. Magn., 36 (4), 861867.

Clemens, M., and T. Weiland (2002), Magnetic field simulation using conformal FIT formulations. IEEE Trans. Magn., 38 (2), 389-392.

Desbrun, M., A. N. Hirani, and J. E. Marsden (2003), Discrete exterior calculus for variational problems in computer vision and graphics. In Proceedings of the 42nd IEEE Conference on Decision and Control (CDC), volume 5, pages 4902-4907. IEEE Press, Washington, DC.

Desbrun, M., E. Kanso, and Y. Tong (2008), Discrete differential forms for computational modeling. In Discrete differential geometry, volume 38 of Oberwolfach Semin., pages 287-324. Birkhäuser, Basel.

Elcott, S., and P. Schröder (2005), Building your own DEC at home. In SIGGRAPH '05: ACM SIGGRAPH 2005 Courses. ACM Press, New York. doi:10.1145/1198555.1198667.

Erickson, J., D. Guoy, J. Sullivan, and A. Üngör (2005), Building spacetime meshes over arbitrary spatial domains. Engineering with Computers, 20 (4), 342-353. doi:10.1007/s00366-005-0303-0. 
Gross, P. W., and P. R. Kotiuga (2004), Electromagnetic theory and computation: a topological approach, volume 48 of Mathematical Sciences Research Institute Publications. Cambridge University Press, Cambridge.

Haber, E., and U. M. Ascher (2001), Fast finite volume simulation of 3D electromagnetic problems with highly discontinuous coefficients. SIAM J. Sci. Comput., 22 (6), 1943-1961.

Hairer, E., C. Lubich, and G. Wanner (2006), Geometric numerical integration, volume 31 of Springer Series in Computational Mathematics. Springer-Verlag, Berlin, second edition. Structure-preserving algorithms for ordinary differential equations.

Harrison, J. (2005), Ravello lecture notes on geometric calculus-Part I. Preprint. arXiv:math-ph/0501001.

Hirani, A. N. (2003), Discrete exterior calculus. Ph.D. thesis, California Institute of Technology. Available from: http://resolver.caltech.edu/ CaltechETD : etd-05202003-095403.

Kale, K. G., and A. J. Lew (2007), Parallel asynchronous variational integrators. Internat. J. Numer. Methods Engrg., 70 (3), 291-321.

Leok, M. (2004), Foundations of computational geometric mechanics. Ph.D. thesis, California Institute of Technology. Available from: http://resolver. caltech.edu/CaltechETD : etd-03022004-000251.

LeVeque, R. J. (2002), Finite volume methods for hyperbolic problems. Cambridge Texts in Applied Mathematics, Cambridge University Press, Cambridge.

Lew, A., J. E. Marsden, M. Ortiz, and M. West (2003), Asynchronous variational integrators. Arch. Ration. Mech. Anal., 167 (2), 85-146.

Lew, A., J. E. Marsden, M. Ortiz, and M. West (2004), Variational time integrators. Internat. J. Numer. Methods Engrg., 60 (1), 153-212.

Marsden, J. E., G. W. Patrick, and S. Shkoller (1998), Multisymplectic geometry, variational integrators, and nonlinear PDEs. Comm. Math. Phys., 199 (2), 351395.

Marsden, J. E., S. Pekarsky, S. Shkoller, and M. West (2001), Variational methods, multisymplectic geometry and continuum mechanics. J. Geom. Phys., 38 (3-4), 253-284.

Marsden, J. E., and M. West (2001), Discrete mechanics and variational integrators. Acta Numer., 10, 357-514.

Nédélec, J.-C. (1980), Mixed finite elements in $\mathbb{R}^{3}$. Numer. Math., 35 (3), 315-341.

Oliver, M., M. West, and C. Wulff (2004), Approximate momentum conservation for spatial semidiscretizations of semilinear wave equations. Numer. Math., 97 (3), 493-535. 
O'Rourke, J. (1998), Computational geometry in C. Cambridge University Press, Cambridge, second edition.

Patrick, G. W. (2004), Geometric classical field theory. Personal communication. Tarhasaari, T., L. Kettunen, and A. Bossavit (1999), Some realizations of a discrete Hodge operator: a reinterpretation of finite element techniques. IEEE Trans. Magn., 35 (3), 1494-1497.

Thite, S. (2005), Spacetime meshing for discontinuous Galerkin methods. Ph.D. thesis, University of Illinois at Urbana-Champaign.

Wang, K., Weiwei, Y. Tong, M. Desbrun, and P. Schröder (2006), Edge subdivision schemes and the construction of smooth vector fields. In SIGGRAPH '06: ACM SIGGRAPH 2006 Papers, pages 1041-1048. ACM Press, New York. doi:10.1145/1179352.1141991.

Whitney, H. (1957), Geometric integration theory. Princeton University Press, Princeton, NJ.

Wise, D. K. (2006), $p$-form electromagnetism on discrete spacetimes. Classical Quantum Gravity, 23 (17), 5129-5176.

Yee, K. S. (1966), Numerical solution of inital boundary value problems involving Maxwell's equations in isotropic media. IEEE Trans. Ant. Prop., 14 (3), 302-307. Yoshimura, H., and J. E. Marsden (2006), Dirac structures in Lagrangian mechanics. II. Variational structures. J. Geom. Phys., 57 (1), 209-250.

Department of Applied and Computational Mathematics, California Institute of Technology, Pasadena CA 91125

Current address: Department of Mathematics, University of California, San Diego, La Jolla CA 92093-0112

E-mail address: ast ern@math.ucsd.edu

Department of Computer Science, California Institute of Technology, Pasadena Ca 91125

Current address: Department of Computer Science and Engineering, Michigan State University, East Lansing MI 48824

E-mail address: ytong@msu. edu

Department of Computer Science, California Institute of Technology, Pasadena CA 91125

E-mail address: mathieu@calt ech.edu

Control and Dynamical Systems, California Institute of Technology, Pasadena CA 91125

E-mail address: jmarsden@caltech.edu 